\title{
Radio observations of active galactic nuclei with mm-VLBI
}

\author{
B. Boccardi ${ }^{1}$ - T. P. Krichbaum ${ }^{1}$ - E. $\operatorname{Ros}^{1,2,3}$ • \\ J. A. Zensus ${ }^{1}$
}

Received: 29 June 2017 / Published online: 6 November 2017

(C) The Author(s) 2017. This article is an open access publication

\begin{abstract}
Over the past few decades, our knowledge of jets produced by active galactic nuclei (AGN) has greatly progressed thanks to the development of very-long-baseline interferometry (VLBI). Nevertheless, the crucial mechanisms involved in the formation of the plasma flow, as well as those driving its exceptional radiative output up to $\mathrm{TeV}$ energies, remain to be clarified. Most likely, these physical processes take place at short separations from the supermassive black hole, on scales which are inaccessible to VLBI observations at centimeter wavelengths. Due to their high synchrotron opacity, the dense and highly magnetized regions in the vicinity of the central engine can only be penetrated when observing at shorter wavelengths, in the millimeter and sub-millimeter regimes. While this was recognized already in the early days of VLBI, it was not until the very recent years that sensitive VLBI imaging at high frequencies has become possible. Ongoing technical development and wide band observing now provide adequate imaging fidelity to carry out more detailed analyses. In this article, we overview some open questions concerning the physics of AGN jets, and we discuss the impact of mm-VLBI studies. Among the rich set of results produced so far in this frequency regime, we particularly focus on studies performed at $43 \mathrm{GHz}(7 \mathrm{~mm})$ and at $86 \mathrm{GHz}(3 \mathrm{~mm})$. Some of the first findings at $230 \mathrm{GHz}(1 \mathrm{~mm})$ obtained with the Event Horizon Telescope are also presented.
\end{abstract}

B. Boccardi

bboccardi@mpifr-bonn.mpg.de

1 Max-Planck-Institut für Radioastronomie, Auf dem Hügel 69, 53121 Bonn, Germany

2 Observatori Astronòmic, Universitat de València, C. Catedrático José Beltrán 2, 46980 Paterna, València, Spain

3 Departament d'Astronomia i Astrofísica, Universitat de València, C. Dr. Moliner 50, 46100 Burjassot, València, Spain 
Keywords High angular resolution · Jets · Active galaxies

\section{Introduction}

Astrophysical jets count among the most spectacular and powerful objects in the Universe. They are collimated outflows of plasma observed in a variety of astronomical sources-young stellar objects (YSOs), X-ray binaries (XRBs), active galactic nuclei (AGN) and $\gamma$-ray bursts (GRBs) — spanning many order of magnitudes both in the energy domain and in linear scale. Despite the diversity of environments where they can originate, all jets share some common features. Their power source is believed to be the gravitational potential of a compact and accreting central object (Salpeter 1964; Lynden-Bell 1969), whose mass largely determines the scaling properties (Sams et al. 1996; Heinz and Sunyaev 2003; Merloni et al. 2003; Falcke et al. 2004). Moreover, all jets are magnetized, as they are, especially in the radio band, copious emitters of synchrotron radiation. Analyzing the similarities as well as the differences between the classes of jets is crucial for ultimately understanding the formation and propagation of the outflows, and the connection between the accretion properties and the jet activity (e.g., Meier 2003; Belloni 2010).

AGN jets, powered by supermassive black holes at the center of some active galaxies, certainly form the most studied class. These highly collimated outflows, with opening angles of few degrees, propagate often undisturbed up to kiloparsec and sometimes megaparsec distances, and radiate over a broad interval of the electromagnetic spectrum. Most of their total power, varying in the range $10^{43}-10^{48} \mathrm{erg} / \mathrm{s}$ (Ghisellini et al. 2014), is however not radiative, but carried in different forms. Close to the launching site it may be purely electromagnetic, while on larger scales it converts to mostly kinetic as the bulk flow accelerates (e.g., Meier 2012), reaching terminal Lorentz factors of the order of ten (Lister et al. 2016). Ultimately, the energy gets dissipated in the form of radiation, giving rise to irregular structures of diffuse radio emission known as radio lobes, sometimes punctuated by compact hotspots. A beautiful representation of the large-scale morphology in the radio galaxy Hercules A is shown in Fig. 1. Although, according to the unification schemes (e.g., Barthel 1989; Urry and Padovani 1995), all jets are intrinsically elongated and two-sided as in Hercules A, many of the sources we can observe appear highly compact and asymmetric. This is due to strong relativistic and projection effects arising from the close alignment of the jet axis with our line of sight, which make the jet properties even more dramatic, but also more difficult to study.

\subsection{The quest for angular resolution}

Starting in 1963, when Maarten Schmidt revealed the extragalactic nature of the radio source 3C 273 (Schmidt 1963), astronomers have extensively investigated the physical processes which could lead to those tremendous energy outputs. The puzzle became even more complicated when the emission was observed to vary on extremely short timescales $t_{\mathrm{var}}$ (see Rees 1970, and references therein). Based on the light travel time argument, the sizes of the emitting regions $l \leq c \cdot t_{\mathrm{var}}$ (with $c$ indicating the speed of 


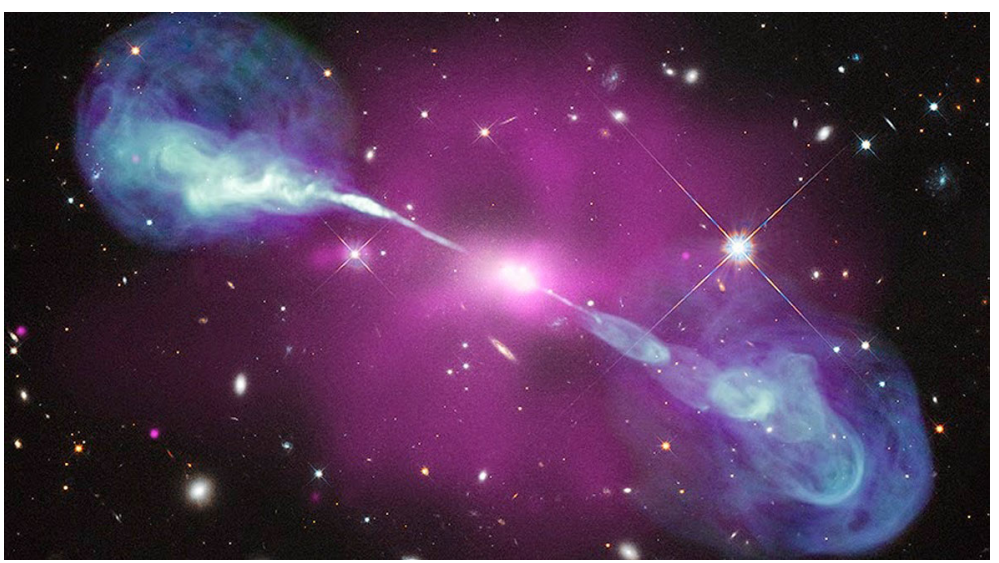

Fig. 1 Composite image of the radio galaxy Hercules A. In blue, the radio emission associated with the jets and the lobes; in pink the X-ray emission from the heated surrounding gas; in white, orange, and blue the host galaxy and the background optical field. Credit: X-ray: NASA/CXC/SAO, Optical: NASA/STScI, Radio: NSF/NRAO/VLA

light) were determined to be as small as few light months. The extreme compactness implied was not only a challenge for theorists, but also for observers. Expressed in angular dimensions, the relevant scales for an AGN jet are in fact of the order of the milli-arcsecond or smaller (for typical distances up to few Giga-parsecs), reaching far beyond the diffraction limit of a single telescope.

Already in the 1940s, radio astronomers had been looking for smart solutions aimed at increasing the resolving power of their instruments. This goal was brilliantly achieved with the development of aperture synthesis (Ryle and Vonberg 1946; Ryle and Hewish 1960), acknowledged by the first Nobel prize for astronomical research, in 1974. Aperture synthesis is an interferometric technique aimed at synthesizing a very large effective aperture from an array of telescopes. This elegant method is applied in its utmost complexity in very-long-baseline interferometry (VLBI), in which the astronomical signal collected by physically unconnected telescopes can be combined. The angular resolution of an interferometer is proportional to the ratio $\lambda / b_{\max }$, where $\lambda$ is the wavelength of the radiation and $b_{\max }$ is the maximum baseline length, i.e., the maximum (projected) separation between two elements of the array. The major VLBI arrays currently in use, the Very Long Baseline Array ${ }^{1}$ (VLBA) and the European VLBI Network (EVN), are characterized by maximum baselines of the order of $10000 \mathrm{~km}$, and perform most of their observations at centimeter wavelengths. Their operation is crucial in the investigation of compact radio sources, and has advanced significantly our knowledge of the physical conditions of the plasma flow on milliarcsecond scales, i.e., on projected spatial extents of the order of few parsecs.

However, the quest for a deeper understanding of these objects is not over. Based on observational and theoretical grounds, the fundamental mechanisms involved in the energy production and in the very formation of the jet outflow are expected to take place

\footnotetext{
1 After recent structural changes within the National Radio Astronomy Observatory (NRAO), the VLBA is now an independent facility operated by the Long Baseline Observatory (LBO-https://www.lbo.us).
} 
on even smaller scales, comparable to the Schwarzschild radius $R_{\mathrm{S}}=2 G M_{\mathrm{BH}} / c^{2}(G$ is the gravitational constant and $M_{\mathrm{BH}}$ is the black hole mass) of the supermassive black hole. Radio interferometric observations capable of probing such scales are, therefore, decisive for obtaining a complete picture of the AGN phenomenon, particularly if used in synergy with high-frequency studies, in the optical, X-ray, and $\gamma$-ray bands (e.g., Marscher 2005).

When aiming at improving the angular resolution of a radio interferometer, two main approaches can be followed. The first consists in increasing the maximum baseline by having one or more telescopes orbiting in space. After some pioneer experiments performed in the 1980s, space-VLBI was successfully realized with the VLBI Space Observatory Program (VSOP) (Hirabayashi et al. 2000) and, more recently, with the RadioAstron mission (Kardashev et al. 2013). In the second approach, ground telescopes can be equipped with receivers operating at shorter wavelengths, in the millimeter or sub-millimeter bands. Both methods enable resolutions of a few tens of micro-arcseconds, which in the closest objects translate into linear sizes as small as few Schwarzschild radii. However, since the nuclear environment is dense and highly magnetized, a truly sharp view of radio cores in AGN can only be obtained by penetrating the opacity barrier shrouding them. Both synchrotron and free-free opacity can affect significantly the cm-wave emission, but are expected to be much reduced in the millimeter band. Millimeter VLBI combines in a unique manner a high spatial resolution with a spectral domain where source-intrinsic absorption effects vanish, and is therefore ideally suited for the imaging of the still unexplored regions in the vicinity of the black hole.

In this article, we discuss some open questions concerning the physics of compact radio sources, and the impact of mm-VLBI observations towards a more detailed physical understanding. In Sect. 2, we briefly summarize the historical development of this sophisticated technique and we report on the capabilities of current mm-VLBI arrays. Section 3 is intended to provide the reader with an overview of the basic properties of jets as inferred from VLBI studies, and of the theoretical models that best describe them. In Sect. 4, we expand the discussion on the scientific topics that mmVLBI can address and we present the main results produced so far. We conclude with an outlook of the future developments and goals of the mm-VLBI science (Sect. 5).

\section{Millimeter-VLBI arrays}

\subsection{General concepts}

Very-long-baseline interferometry (VLBI) is an elegant observing technique which, as of this writing, provides the highest possible angular resolution in astronomy (see Gómez et al. 2016, for the highest resolution image produced to date). In the following part of this section, we will detail the capabilities of VLBI arrays operating in the millimeter regime. First, however, we wish to introduce some necessary terminology and fundamental parameters which define the performance of radio telescopes and interferometers. The following description is by no means complete, and the reader 
is referred to the specialized textbooks on interferometry and synthesis imaging (e.g., Thompson et al. 2017).

A radio interferometer is an instrument which enables to combine the radio waves coming from an astronomical object to form interference fringes. By correlating the signals collected simultaneously by each telescope forming the array, radio interferometers measure the complex visibility function $V(u, v)$, which is the (noise-corrupted) Fourier transform of the brightness distribution of the sky. The $(u, v)$ coordinates define, in units of wavelength, the East-West and the North-South component of each baseline projected in the sky, as seen from the source. Thus, the $(u, v)$-plane contains information about the existence or absence of a visibility measurement in a certain point. The filling of the $(u, v)$-plane, i.e., the $(u, v)$-coverage, is by definition incomplete for any interferometer, and can be improved by adding more telescopes to the array and by increasing the on-source time up to $12 \mathrm{~h}$, so that the Earth rotation enables a single baseline to sample a full track (an ellipse) in the $(u, v)$-plane. Each baseline of projected length $b$ is characterized by its own fringe pattern, and is only sensitive to source structures on scales comparable to the fringe spacing $\lambda / b$. Therefore, the better the sampling of the $(u, v)$-plane, the more reliable will be the reconstruction of the sky brightness distribution. The smallest angular scale an interferometer can probe, its resolution, coincides, in general, with the diffraction limit $\sim \lambda / b_{\max }$, where $b_{\max }$ is the maximum baseline length.

While the angular resolution achieved at a given wavelength depends, in principle, only on the geometrical properties of the array, the sensitivity of interferometric observations is largely dependent on the characteristics of the single telescopes forming the array and of the recording systems in use. Given an array formed by $N$ telescopes, the sensitivity of a single telescope with geometrical area $A$ is quantified by the system equivalent flux density SEFD, a parameter which accounts for the combined sensitivity of the antenna and of the receiving system. The SEFD [Jy] is given by the ratio $T_{\text {sys }} / g$, where the system temperature $T_{\text {sys }}[\mathrm{K}]$ is the sum of all the noise contributions, both from the electronics and from the astronomical source, while the gain $g$ $[\mathrm{K} / \mathrm{Jy}]$ quantifies the radio noise power received from a source of unit flux density. The gain is a measure of the antenna efficiency $\eta$, an elevation-dependent parameter which determines the effective collective area $A_{\mathrm{eff}}=\eta A$ of the dish. At short radio wavelengths, the efficiency is especially limited by the antenna surface accuracy. For instance, a surface accuracy $s=\lambda / 16$ implies a reduction of the effective collecting area by a factor $\eta_{s}=\exp (-4 \pi s / \lambda)^{2} \simeq 0.5$.

The astronomical signal is extremely weak with respect to the noise produced by the receiving system, and the signal-to-noise ratio must then be increased by averaging over a large number of samples $n_{\mathrm{s}}$. However, the number of samples cannot be arbitrarily high, being limited by the bandwidth of the signal $\Delta v$. According to the Nyquist sampling theorem, samples taken over time intervals $\Delta t$ shorter than $1 / 2 \Delta v$ are not independent. Therefore, the number of samples $n_{\mathrm{s}}=2 \Delta v t$ has to be increased by increasing the observing time $t$ and/or by extending the observing bandwidth $\Delta v$. According to the central limit theorem, averaging over a large number of samples will reduce the rms noise $\sigma$ by a factor of $\sqrt{n_{\mathrm{s}}}$, which allows us to write the radiometer equation as: 


$$
\sigma=\frac{\sqrt{2} T_{\mathrm{sys}}}{\sqrt{2 \Delta \nu t}}=\frac{T_{\text {sys }}}{\sqrt{\Delta \nu t}} .
$$

Among the possible ways to achieve a higher sensitivity, increasing the bandwidth is the most cost-effective. For this reason, the technical improvements in VLBI, and in radio interferometry in general, have been especially oriented towards the development of wide bandwidth receivers and recording systems.

In VLBI mode, radio signals are digitized and recorded at each telescope on magnetic tapes, together with extremely precise time stamps usually provided by an hydrogen maser clock. The digitization process has also an impact on sensitivity, and reduces the signal to noise ratio by a factor $\eta_{\mathrm{c}} \leq 1$, called VLBI system efficiency, with respect to an ideal analog recording. The VLBI system efficiency depends on the number of bits used to represent each sample, with $\eta_{\mathrm{c}} \sim 0.63$ for a 1 bit representation and $\eta_{\mathrm{c}} \sim 0.87$ for a 2 bit representation. The sensitivity provided by a certain digital recording system is, therefore, determined by the data rate, expressed in bit/s.

Ultimately, the theoretical thermal noise $\sigma_{\text {im }}$ of a VLBI image depends both on the SEFD of each telescope and on the data rate, and can be expressed in units of Jy/beam as:

$$
\sigma_{\mathrm{im}}=\frac{1}{\eta_{\mathrm{c}}} \frac{\mathrm{SEFD}^{*}}{\sqrt{N(N-1) \Delta v t_{\mathrm{int}}}},
$$

where $\mathrm{SEFD}^{*}=\sqrt{\sum_{i, j=1}^{N ; i<j}\left(\mathrm{SEFD}^{i} \times \mathrm{SEFD}^{j}\right)}$ and $t_{\text {int }}$ is the total integration time on source in seconds.

\subsection{A brief history and current status}

The possibility of conducting VLBI experiments at millimeter wavelengths was first explored in the late 1970s. Only a few years later, in the early 1980s, pioneering experiments were successfully performed through the observation of the strong radio source 3C 84 at $86 \mathrm{GHz}$ (3 mm) (Readhead et al. 1983) and $43 \mathrm{GHz}$ (7 mm) (Marcaide et al. 1985). The choice of these observing bands was naturally determined by the behavior of atmospheric transmission in the radio window. As shown in Fig. 2, strong opacity spikes due to molecular absorption in the troposphere (mainly from water vapor and oxygen) overlay the general trend of steeply increasing opacity as a function of frequency, and bracket three main observing windows at around 35, 90, and $135 \mathrm{GHz}$.

Since those first detections, the performance of mm-VLBI arrays has been steadily improving, until a sensitive imaging (with dynamic range $>1000$, e.g., Boccardi et al. 2016b) has become possible. The road through the technical development has been challenging, and sometimes rough. The main limitation in interferometric experiments at millimeter wavelengths is the reduced phase stability, resulting in coherence losses. In VLBI experiments, phase fluctuations can result from the contribution of several factors, e.g., inaccuracies in the hydrogen maser frequency standards or in the local oscillator systems of the receiver, antenna-pointing errors, uncertainties in the antennas positions (see, e.g., Rogers and Moran 1981). At high radio frequencies, however, phase drifts are mostly of atmospheric origin (see Rogers et al. 1984, for a discussion), being primarily caused by water vapor in the troposphere. High altitude and/or dry 


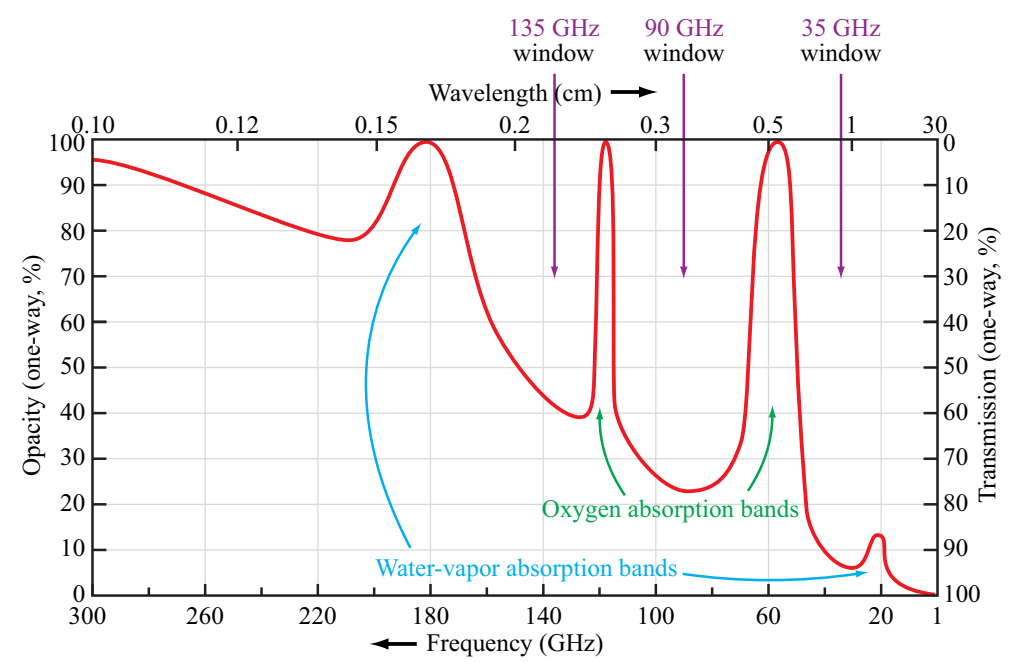

Fig. 2 Percentage transmission through the Earth's atmosphere, in the vertical direction, under clear sky conditions (Ulaby et al. 2014). Strong molecular absorption is caused by the water vapor and the oxygen in the troposphere

sites are the optimal choice for minimizing the degradation of the signal in such observations. While excellent instruments, like the Plateau de Bure interferometer or the Atacama Large Millimeter Array (ALMA), were built ad hoc in optimal sites in the following years, initial millimeter arrays could only count on the existing telescopes, most of which were conceived for operating at centimeter-waves. This, combined with the poor $(u, v)$-coverage, limited bandwidths and inaccurate calibration procedures, has severely hindered the imaging capabilities of past mm-VLBI arrays.

An important step forward was made with the foundation of global arrays operating at $86 \mathrm{GHz}$, the Coordinated Millimeter VLBI Array (CMVA) (Rogers et al. 1995), established in 1995, and its successor, the Global Millimeter VLBI Array (GMVA) ${ }^{2}$ (Krichbaum et al. 2006), whose activity is ongoing since 2003. The participation of a larger number of telescopes $(>10)$, the inclusion of sensitive antennas like the IRAM telescopes (Pico Veleta and the phased Plateau de Bure) or the Effelsberg $100-\mathrm{m}$, and the remarkable increase in bandwidth (by a factor $>10$ ) gradually led to an improvement of the performance. This fact can be immediately recognized when considering the detection rate of $86 \mathrm{GHz}$ VLBI surveys conducted between 1997 and 2008. This increased from a percentage of less than $25 \%$ obtained in the first campaigns (Beasley et al. 1997; Lonsdale et al. 1998; Rantakyrö et al. 1998) to more than $90 \%$ achieved in the latest (Lobanov et al. 2000; Lee et al. 2008; Gopalakrishnan Nair et al. 2016).

Currently, the GMVA comprises up to 18 telescopes (Effelsberg, Onsala, Plateau de Bure, Pico Veleta, Yebes, Metsähovi, Green Bank, eight VLBA stations and the three telescopes forming the Korean VLBI Network-KVN) spread over three continents

2 http://www3.mpifr-bonn.mpg.de/div/vlbi/globalmm/. 
Table 1 Properties of the antennas operating at $86 \mathrm{GHz}$

\begin{tabular}{llrr}
\hline Station & Location & Effective diameter [m] & SEFD [Jy] \\
\hline Effelsberg & Germany & 80 & 1000 \\
Onsala & Sweden & 20 & 5100 \\
Plateau de Bure & France & 34 & 820 \\
Pico Veleta & Spain & 30 & 650 \\
Yebes & Spain & 40 & 1700 \\
Metsähovi & Finland & 14 & 17000 \\
Green Bank & United States & 100 & 140 \\
VLBA $(\times 8)$ & United States & 25 & 2500 \\
KVN $(\times 3)$ & South Korea & 21 & 3200 \\
ALMA & Chile & 85 & 60 \\
\hline
\end{tabular}

Col. 1: Name of the telescope. Col. 2: Its location. Col. 3: Effective diameter in meters. Col. 4: Typical value of the system equivalent flux density (SEFD), which is a measurement of the telescope sensitivity. Lower values indicate a better performance

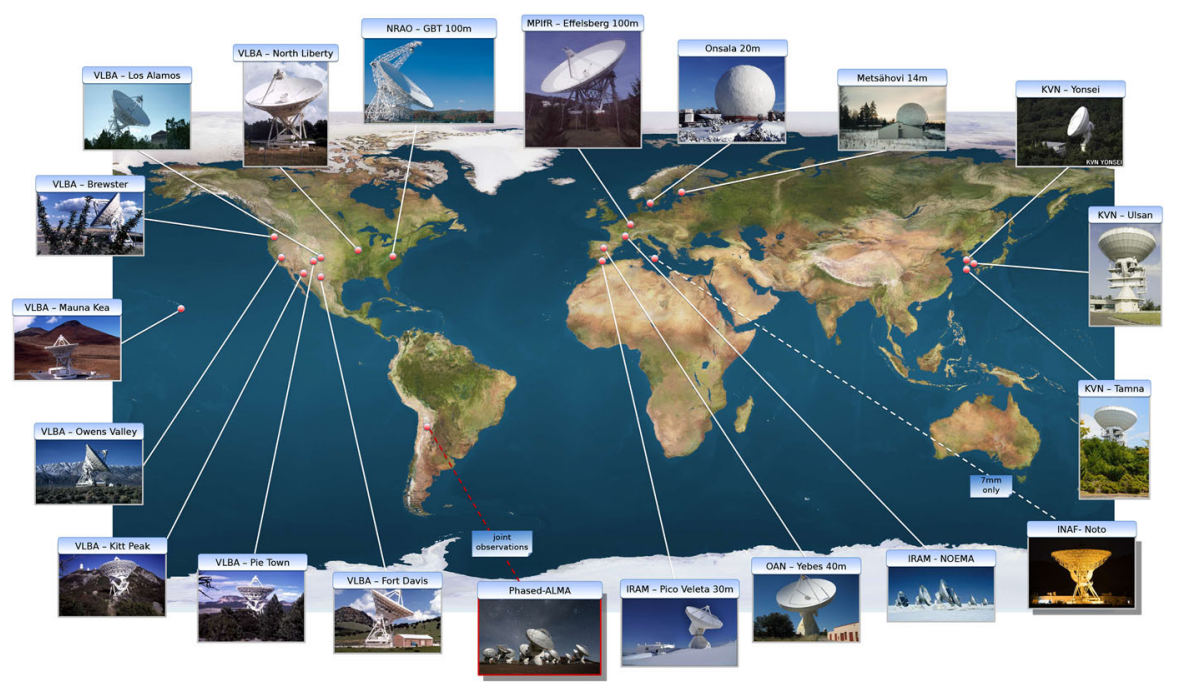

Fig. 3 The telescopes forming the Global Millimeter VLBI Array (GMVA), operating at the frequency of $86 \mathrm{GHz}$. Credit: Helge Rottmann

(Europe, America, and Asia-Table 1, Fig. 3). The phased ALMA, located in Chile, has also participated for the first time in mm-VLBI experiments in April 2017. To date, most of the GMVA stations are observing at a bandwidth of $512 \mathrm{MHz}$ (which corresponds to a data rate of $2048 \mathrm{Mbit} / \mathrm{s}$ ), and further upgrades are expected in the near future thanks to the fast development of digital VLBI recording systems. Global VLBI observations at $86 \mathrm{GHz}$ can achieve a typical angular resolution of 50-70 microarcseconds, and an array sensitivity of $\sim 0.9 \mathrm{mJy} / \mathrm{h}$.

At frequencies higher than $86 \mathrm{GHz}$, atmospheric effects become even more dominant, and VLBI experiments are plagued with additional difficulties. Nevertheless, 
fundamental science goals (Sect. 4.5) have driven the technical development also in this frequency regime. A first experiment at $223 \mathrm{GHz}(1.4 \mathrm{~mm})$ was successfully performed already in 1989 (Padin et al. 1990), with a single-baseline fringe detection of 3C273. Subsequent observations involving telescopes like Pico Veleta, Plateau de Bure, CARMA (Combined Array for Research in Millimeter-Wave Astronomy), the SMTO (Sub-millimeter telescope) and, since 2012, also APEX (Atacama Pathfinder Experiment) confirmed the feasibility of 1mm-VLBI (e.g., (Krichbaum et al. 1997, 1998; Doeleman et al. 2008; Wagner et al. 2015), achieving the detection of several other sources also on transatlantic baselines. Much of the development of $1 \mathrm{~mm}-\mathrm{VLBI}$ is driven at present by the Event Horizon Telescope $(\mathrm{EHT})^{3}$ project. When completed, the EHT will be able resolve the black hole surroundings on scales comparable with the event horizon. The expected resolution is of 20-30 $\mu$ as at $230 \mathrm{GHz}$ and of 13-20 $\mu$ as at $345 \mathrm{GHz}$. Due to the large apparent size of their event horizon, Sagittarius A* and M87 are the best candidates for achieving such a goal and, therefore, are considered as primary targets for EHT observations.

\section{Extragalactic jets as seen by VLBI}

In VLBI images, the radio emission from an AGN can be typically ascribed to a compact, bright, and unresolved feature called the "core", and to a one-sided jet emanating from it (Fig. 4). This classical morphology is the result of selection effects, involving both the relativistic nature of the flow and the sensitivity of VLBI arrays.

Similarly to Hercules A (Fig. 1), all jets are thought to be characterized by a roughly symmetric two-sided structure. However, due to the relativistic nature of the flow, the emission from the side approaching the observer can be highly enhanced as a consequence of relativistic Doppler boosting. By applying the Lorentz transformations, it can be shown that the observed flux density $S_{\mathrm{o}}$ differs from the intrinsic one $S_{\mathrm{e}}$ by a factor $\delta^{n-\alpha}$, where $\delta$ is the Doppler factor, $\alpha$ is the spectral index, and $n$ is a parameter varying between 2 and 3 (see Scheuer and Readhead 1979). The Doppler factor depends on the speed of the flow $\beta=v / c$, which determines the bulk Lorentz factor $\Gamma=1 / \sqrt{\left(1-\beta^{2}\right)}$, and on the jet orientation, defined by the angle $\theta$ between the direction of propagation of the outflow and the line of sight of the observer:

$$
\delta=\frac{1}{\Gamma(1-\beta \cos \theta)} .
$$

Given a population of jets oriented randomly and spanning a certain range of Lorentz factors, it is clear from Eq. 3 that Doppler boosting increases the chances to detect the fastest objects and/or those oriented at small angles (naturally, there is also a dependence on the intrinsic luminosity function of the jet population, see, e.g. Lister and Marscher 1997). The frequent observation of superluminal motion, i.e., of apparently faster-than-light speeds $\beta_{\text {app }}$ of the plasma features in the jet confirms the selection bias affecting the jet samples. This purely geometrical phenomenon, predicted by Rees

\footnotetext{
3 http://www.eventhorizontelescope.org/.
} 


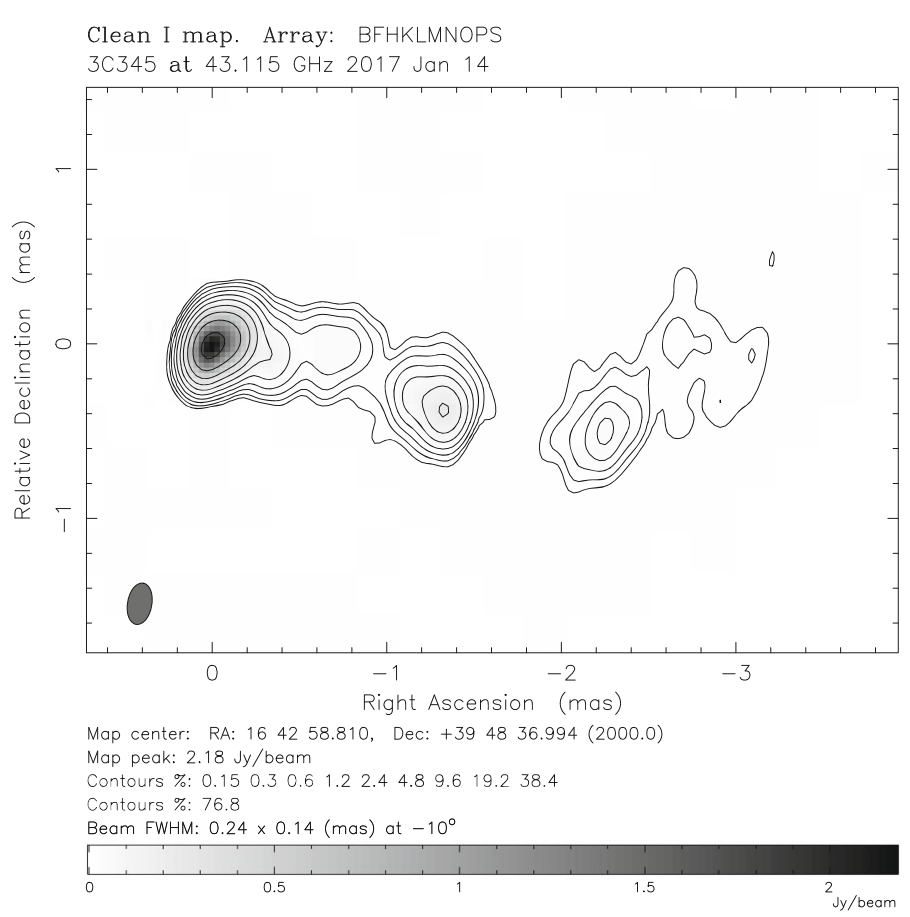

Fig. 4 A $43 \mathrm{GHz}$ image of the blazar 3C 345 from VLBA observations in January 2017. The source shows a typical core-jet structure, with bright knots punctuating the jet emission. The image was produced by the authors from the data available at the public archive of the VLBA-BU Blazar Monitoring Program (VLBA-BU-BLAZAR; http://www.bu.edu/blazars/VLBAproject.html)

(1966) and observed for the first time in 3C 273 (Gubbay et al. 1969), occurs more prominently in fast flows seen at small angles, as described by the relation

$$
\beta_{\text {app }}=\frac{\beta \sin \theta}{1-\beta \cos \theta} .
$$

Another selection effect in VLBI is related to sensitivity. It is not sufficient for an astronomical source to be a strong radio emitter to be seen by VLBI. Only "high intensity" objects, i.e., with high brightness temperature $T_{\mathrm{b}}$, are suitable targets. The minimum brightness temperature $T_{\mathrm{b}}^{\mathrm{min}}$ detectable by an interferometer depends on the flux density $S_{v}$, the baseline length $b$, and the Boltzmann constant $k$ as

$$
T_{\mathrm{b}}^{\min }=\frac{2}{\pi k} b^{2} S_{v}
$$

and is typically in the range $10^{6}-10^{8} \mathrm{~K}$. The latter implies that both thermal emission $\left(T_{\mathrm{B}}<10^{5} \mathrm{~K}\right)$ and non-thermal emission of insufficient compactness (e.g., from the radio lobes) are completely resolved out in a VLBI observation. In conclusion, the samples targeted by VLBI are dominated by the highly boosted compact objects, also 
known as blazars. ${ }^{4}$ While studies of blazars may provide an incomplete view of the general jet phenomenon in $\mathrm{AGN}$, these sources represent a unique cosmic laboratory for investigating the physics of relativistic plasmas in extreme conditions.

\subsection{Where do we stand?}

The physical conditions of the plasma forming a jet evolve significantly as the jet propagates from the central engine through the medium. According to the current paradigm, it is possible to identify four distinct regions (Fig. 5): launching, acceleration and collimation, kinetic-flux dominated, and dissipation.

Jets are thought to be launched in the immediate vicinity of the supermassive black hole, at distances of $\leq 10^{2} R_{\mathrm{S}}$ (Meier et al. 2001). In the launching region, the plasma is channeled through the action of strong magnetic fields, which extract part of the energy stored in the accretion disk (Blandford and Payne 1982) and/or in the rotating black hole (Blandford and Znajek 1977). The composition of the loaded matter is not well determined: the jet may consist of a normal electron-proton plasma (e.g., Celotti and Fabian 1993) or it may be formed by light particles only, electrons and positrons (e.g., Reynolds et al. 1996).

This purely electromagnetic flow is then accelerated and collimated. In the second region, reaching up to scales of $10^{3}-10^{5} R_{\mathrm{S}}$ ( $\sim$ sub-parsec to parsec) (Vlahakis and Königl 2003, 2004), the initial broad stream is rapidly focused thanks to the confinement provided by the magnetic field and/or by the external medium, and is accelerated to relativistic speeds by magnetic pressure gradients (e.g., Spruit et al. 1997; Komissarov et al. 2007; Lyubarsky 2009). At the end of this process, a large part of the magnetic energy has been converted to kinetic energy. The kinetic-flux dominated jet extends between $10^{5}-10^{9} R_{\mathrm{S}}$ (parsecs to kiloparsecs). In this region, the magnetic field is expected to become dynamically unimportant, and the jet can be appropriately described by the physical laws of gas dynamics (Daly and Marscher 1988). Being subject to hydrodynamic shocks (Blandford and Königl 1979; Marscher and Gear 1985) and plasma instability (Blake 1972; Hardee 1979), the flow ultimately looses its collimation and dissipates its energy in the form of radiation. Within the lobes formed at distances $\geq 10^{9} R_{\mathrm{S}}$ ( $>$ kiloparsecs), compact hotspots are often observed, suggesting that part of the plasma can still be highly relativistic on large scales (Georganopoulos and Kazanas 2004).

Over the past 40 years, radio interferometric observations have provided us with a detailed description of the two outermost regions. In particular, VLBI studies at centimeter wavelengths have significantly improved our understanding of parsec-scale flows (also known as compact jets). Both statistical studies of large samples-first among all, those performed at $2 \mathrm{~cm}$ within the MOJAVE program $^{5}$ (Lister et al. 2009) -

\footnotetext{
4 According to the unification schemes (Urry and Padovani 1995), blazars come in two flavors: FSRQs (Flat Spectrum Radio Quasars), which show broad emission lines and powerful, highly collimated jets, and BL Lacs, which lack emission or absorption features and have less powerful jets. The parent population of jets oriented at larger viewing angles is formed by the radio galaxies, Fanaroff-Riley II and Fanaroff-Riley I, respectively (Fanaroff and Riley 1974).

5 http://www.physics.purdue.edu/astro/MOJAVE/.
} 


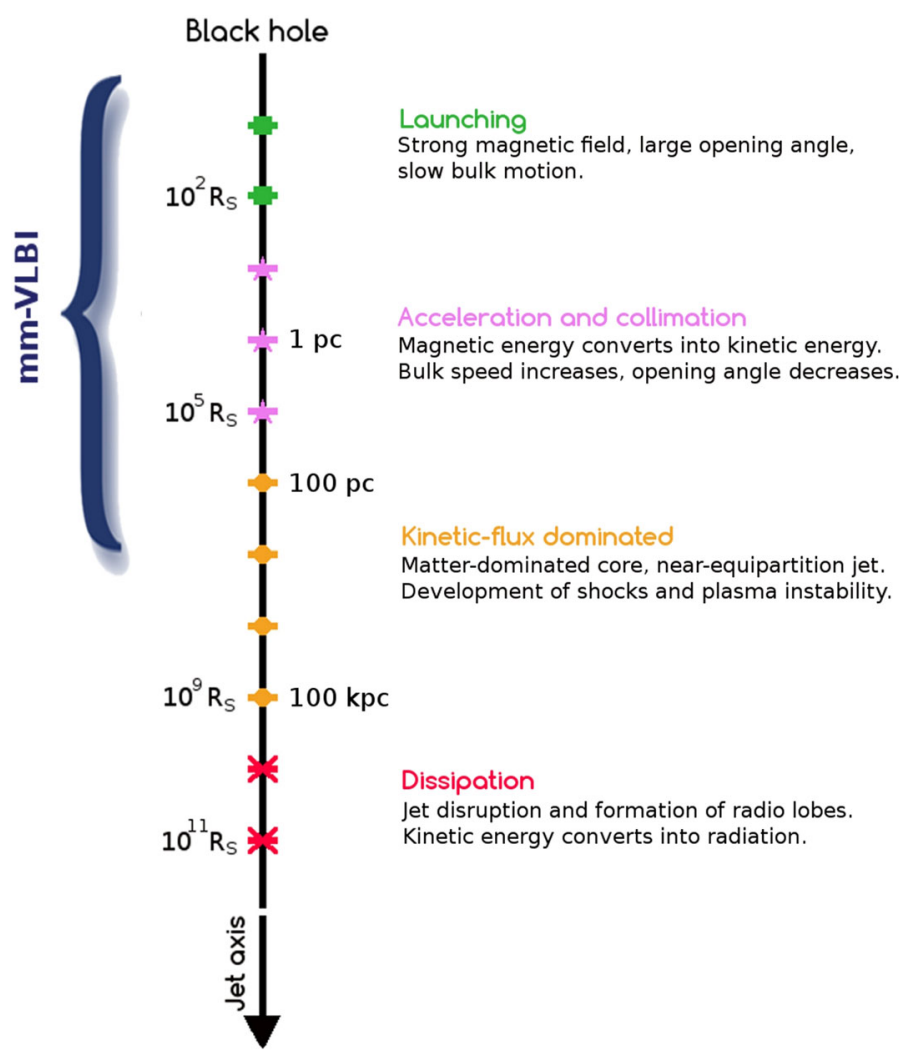

Fig. 5 Schematic view of the main regions of a relativistic jet, according to the current paradigm for magnetically-driven flows. The radial separation from the black hole is represented in a logarithmic scale, in units of Schwarzschild radii $R_{\mathrm{S}}$. We also report the corresponding distance in units of parsecs (pc) for a black hole mass of $10^{9} M_{\odot}$. The extension of each region is approximate, and may vary in different jets. VLBI observations at millimeter wavelengths are suited for probing the magnetically dominated jet base and the transition to the kinetic-flux dominated region

and detailed analyses of single targets were able to address several key topics. How fast does the plasma flow? What is the energy balance between the magnetic field and the particles? Is the magnetic field ordered? What are jets made of? And what is that we really observe with VLBI? Providing a complete summary of these results is a challenging task, and beyond the scope of this review. The interested reader is referred to the review articles from Zensus (1997) and Marscher (2006). In the following, we discuss some highlights, with the aim of better contextualizing the scientific questions that are more relevant for mm-VLBI studies.

Centimeter VLBI imaging has shown that a large fraction of the parsec-scale emission is produced by the core component, the upstream feature from which the jet appears to emanate. The radio core, characterized by a flat spectrum and weak polarization and usually located at parsec-distances from the black hole, is classically interpreted as marking the transition between synchrotron self-absorbed regions and optically thin regions (Blandford and Königl 1979; Königl 1981). The flatness of 
the spectrum is explained as the result of the superposition of different synchrotron self-absorbed components in a conical geometry (see also Marscher 1996). In this scenario, the synchrotron opacity associated with the core component implies that its position is not fixed, but has a frequency dependence. Specifically, the emission should be detected at progressively smaller distances from the central engine with increasing observing frequency. This effect, known as core-shift, is observed in many jets (Marcaide and Shapiro 1984; Lobanov 1998), although not in all (Pushkarev et al. 2012).

The emission from the jet is largely ascribed to the development of relativistic hydrodynamic shocks and plasma instability. The Kelvin-Helmholtz instability (Blandford and Pringle 1976; Turland and Scheuer 1976; Ferrari et al. 1978), formed in the presence of velocities shears between two fluids, is likely to dominate the emission and shape the flow on hectoparsec and larger scales (Hardee 2000; Lobanov and Zensus 2001). Shocks, instead, are a distinctive feature of parsec-scale flows (Blandford and Königl 1979; Marscher and Gear 1985). Indeed, most of the jets imaged with VLBI do not appear as continuous flows, but can be well modeled as a sum of discrete features, known as blobs or knots. Shocks originate either from pressure mismatches at the boundaries with the external medium or from major changes at the base of the flow (e.g., new plasma ejections or directional changes). They are sites of efficient particle acceleration through the Fermi mechanism (e.g., Bell 1978) and of local amplification of the magnetic field. Accordingly, strong variability of the emission and enhanced polarization can often be associated with shocked regions in VLBI images of jets. Shocks are observed to move superluminally, with apparent speeds as high as $\sim 50 c$, but more commonly below $10 c$ (Lister et al. 2016).

In addition to moving features, jets are also punctuated by bright stationary spots (e.g., Kellermann et al. 2004; Lister et al. 2009; Agudo et al. 2012a). These have generally been interpreted as standing shocks, formed, e.g., as a consequence of prominent recollimation events (e.g., Daly and Marscher 1988; Gomez et al. 1995). Stationary features in the vicinity of the jet base are thought to play an important role for the production of non-thermal high-energy emission in AGN (e.g., León-Tavares et al. 2010; Arshakian et al. 2010), and may mark fundamental transition regions of the jet. For instance, the presence of a recollimation shock may be expected at the end of the acceleration and collimation region, when the jet becomes causally disconnected from the central engine (e.g., Polko et al. 2010; Cohen et al. 2014).

In some objects, particularly in those not showing an appreciable core-shift, the VLBI core itself may coincide with such a recollimation feature or, more generally, with the first and brightest standing shock developing in the flow (Marscher 2008, 2009). The nature of the radio core, still debated, is clearly a crucial element to understand, and much of the recent theoretical work has been aimed at identifying the dissipative events which could account for the broadband emission, variability and polarization properties observed in its vicinity (e.g., Sikora et al. 2009; Sironi et al. 2015, and references therein). Radio observations indicate that, whichever mechanism is taking place, it must lead to very efficient energy dissipation. This is inferred, for instance, from measurements of the brightness temperature of individual components in VLBI images, obtained after estimating their flux density $S_{v}$ and angular dimension $d$ as: 


$$
T_{\mathrm{b}}=1.22 \times 10^{12} \frac{S_{v}(1+z)}{d^{2} v^{2}} .
$$

At centimeter wavelengths, the intrinsic brightness temperature $T_{0}=T_{\mathrm{b}} / \delta$ of the core reaches (Kellermann et al. 1998; Homan et al. 2006) and sometimes exceeds (Kovalev et al. 2016) the maximum theoretical value expected for a synchrotron self-absorbed component. This limit is posed by the onset of the inverse Compton catastrophe, i.e., by the strong enhancement of the inverse Compton cooling in a synchrotron-emitting region whose temperature has approached a threshold of $\sim 5 \times 10^{11} \mathrm{~K}$ (Kellermann and Pauliny-Toth 1969). High brightness temperatures are especially measured during flares, and indicate that VLBI cores are strongly particle dominated. In the jet, instead, a rapid temperature drop is observed, with values which are usually in very good agreement with the equipartition limit of $\sim 5 \times 10^{10} \mathrm{~K}$ (Readhead 1994), i.e., with the temperature expected when the energy of the radiating particles equals the energy stored in the magnetic field.

The details of the processes taking place between the jet launching region, where the jet is a purely electromagnetic stream, and the matter-dominated cm-VLBI core are not known yet. Decisive constraints can only come from direct observational probes of the relevant spatial scales, which is where mm-VLBI comes into play.

\section{AGN science with mm-VLBI}

In the following part of this review, we focus on the discussion of the main open questions concerning the physics of AGN jets that can be ideally addressed by the use of the mm-VLBI technique. The results presented are mainly from observations at 43 and $86 \mathrm{GHz}$, but include some of the first findings at $230 \mathrm{GHz}$. At $43 \mathrm{GHz}$, an important contribution comes from the blazar monitoring program run by the Boston University ${ }^{6}$ (Jorstad and Marscher 2016) with the VLBA.

We start by describing the possible mechanisms giving rise to the high-energy emission and how mm-VLBI observations can help constraining its location (Sect. 4.1). From this discussion, the importance of probing the internal structure of the flow and its morphological aspects will emerge, a topic which is expanded in Sect. 4.2. We will then address the broad subject of jet formation, investigated through polarization studies (Sect. 4.3) or through the direct imaging of the jet launching region (Sect. 4.4). We will conclude with a short overview of the first scientific results and future goals in the study of the compact radio source in our Galaxy, Sagittarius A*, with the Event Horizon Telescope.

\subsection{High-energy emission}

Since the launch of CGRO/EGRET (Compton Gamma-Ray Observatory/Energetic Gamma-Ray Experiment Telescope, Thompson et al. 1993), AGILE (Astro-rivelatore Gamma a Immagini Leggero, Tavani et al. 2008), and especially of the Fermi/LAT

\footnotetext{
6 https://www.bu.edu/blazars/VLBAproject.html.
} 
(Large Area Telescope, Atwood et al. 2009), blazars have been established as the most numerous class of $\gamma$-ray sources in the sky. To date, Fermi has identified over 1000 blazars (Acero et al. 2015), a fraction of which is also detected at TeV energies. ${ }^{7}$

The most frequently invoked process to account for the high-energy emission observed in AGN is the inverse Compton (IC) scattering of soft photons by the population of relativistic electrons forming the jet. In this scenario, the electrons are giving rise to both the low-frequency synchrotron component and the high-frequency bump characterizing the spectral energy distribution (SED). In principle, the reservoir of soft photons can originate in various regions of the AGN. In the synchrotron self-Compton mechanism, the same synchrotron photons emitted by the jet are up-scattered to higher energies (Maraschi et al. 1992). Concerning the "external" reservoirs, instead, several possibilities have been proposed. In order of increasing distance from the central engine, these include optical/UV photons from the accretion disk (Dermer and Schlickeiser 1993) and from the broad line region (Sikora et al. 1994), infra-red emission from the torus (Błażejowski et al. 2000), and, on larger scales, CMB photons (e.g.,Celotti et al. 2001). Alternatively, if ultra-relativistic protons are also present in the jet, the $\gamma$-ray emission may result from proton-synchrotron or from $p \gamma$ photopion production (Mannheim and Biermann 1992; Aharonian 2000). Especially in hadronic models, but also in the leptonic ones, extremely efficient acceleration mechanisms are required and must be at play in AGN jets.

Statistical studies of large samples (Ackermann et al. 2011; Fuhrmann et al. 2014; Ramakrishnan et al. 2015) have inferred the existence of significant correlations between the broadband $(0.1 \mathrm{GeV}<E<300 \mathrm{GeV})$ Fermi light curves and the radio ones obtained from single-dish monitoring programs at centimeter and millimeter wavelengths (see, e.g., the F-GAMMA legacy Program) ${ }^{8}$, suggesting a common origin of the emission in the two bands. In the aforementioned studies, the radio variability is usually found to be delayed with respect to the high frequencies. Similar conclusions were reached through a VLBI analysis of the MOJAVE sample (Pushkarev et al. 2010) showing, in addition, that the radio- $\gamma$ correlations are highly significant when considering the radio properties of the core, rather than those of the jet.

From a theoretical standpoint, the observed behavior may be conveniently explained if the activity-triggered, for instance, by a prominent change in particle density of the plasma-arises in a synchrotron self-absorbed region located in the innermost surroundings of the central engine. Here, highly relativistic electrons in the jet can up-scatter the optical/UV photons from the broad-line region to $\gamma$-ray energies. At energies above $1 \mathrm{GeV}$, this dense photon-field is also expected to be highly opaque to the emerging $\gamma$-rays through the reaction $\gamma \gamma \rightarrow e^{ \pm}$(see, e.g., Ghisellini and Tavecchio 2009), which could account for the GeV spectral break observed in several blazars (Poutanen and Stern 2010). Moreover, one of the main arguments in favor of the "close dissipation" scenario is given by variability timescales as short as few hours, or even minutes (e.g., Aharonian et al. 2007; Ackermann et al. 2016), which constrain the size of the emitting regions involved to be highly compact

\footnotetext{
7 http://www.tevcat.uchicago.edu.

8 http://www3.mpifr-bonn.mpg.de/div/vlbi/fgamma/.
} 
and have led to the conclusion that $\gamma$-rays must originate within $10^{16} \mathrm{~cm}$, i.e., $\ll 1 \mathrm{pc}$, from the central engine (Tavecchio et al. 2010).

It has also been argued, however, that assuming a direct correspondence between the inferred size of the region and its distance from the central engine may be incorrect (e.g., Marscher and Jorstad 2010). While showing very rapid variability, the highenergy emission may still be produced at larger separations from the jet base if the emission region occupies only a small fraction of the jet cross-section.

Based on the results from the Metsähovi monitoring at 22 and $37 \mathrm{GHz}$ of the EGRET-detected blazars, Valtaoja and Terasranta (1995) were among the first to propose that, especially in quasars, the $\gamma$-ray flares are likely to originate in the millimeter-wave emitting regions (see also Valtaoja and Teraesranta 1996; Lähteenmäki and Valtaoja 2003; León-Tavares et al. 2011) rather than in the vicinity of the broad line region. Thanks to its ability to detect detailed structural changes in the flow, mm-VLBI has now provided compelling evidence that, in fact, the high-energy events and the millimeter-wave emission are often co-spatial, corroborating physical scenarios where the $\gamma$-ray emission is produced at parsec distances from the central engine. Some of the mm-VLBI studies supporting this idea, as well as findings possibly requiring alternative interpretations, are summarized in the following.

Already before the launch of Fermi, a systematic VLBI study of a large sample of $\gamma$ ray blazars detected by EGRET was conducted at frequencies up to $43 \mathrm{GHz}$ by Jorstad et al. (2001). In a large number of sources, a clear connection is observed between the occurrence of a $\gamma$-ray flare and the ejection of a new superluminal component. The $\gamma$ ray flare is found, on average, to follow the ejection, and to nearly coincide with a local maximum in the polarized radio flux density. By interpreting the newly ejected components as traveling shocks, this delay could coincide with the time required for the shock to fully develop and for the electrons to be efficiently accelerated at its forward layer.

More recent VLBI monitoring campaigns of single objects of $43 \mathrm{GHz}$ have confirmed that, in general, a tight relation exists between the $\gamma$-ray emission and the properties of the mm-VLBI core region. In sources like 1156+295 (Ramakrishnan et al. 2014), PKS 1510-089 (Aleksić et al. 2014) and 0954+658 (Morozova et al. 2014), the $\gamma$-ray outbursts are found to be triggered by the passage of new superluminal components through the mm-VLBI core. In the case of 3C 120 (Casadio et al. 2015a) and CTA 102 (Casadio et al. 2015b), this trend is confirmed, but only when the newly ejected features are traveling in a direction closer to the observer's line of sight. Indeed, a close connection between the jet orientation and the occurrence of $\gamma$-ray flares is evident at a statistical level in the MOJAVE survey (Pushkarev et al. 2009), since the class of $\gamma$-ray loud jets is found to be oriented at smaller viewing angles with respect to the general radio-loud population. Flaring events triggered by the ejection of new components are often accompanied by increased activity in the optical and X-rays, and by systematic rotations of the optical electric vector position angle (e.g., Marscher et al. 2008; Jorstad et al. 2010; Marscher et al. 2010).

In several cases, the mm-core where the high-energy emission is produced has been identified as a standing shock (e.g., D'Arcangelo et al. 2007; Marscher et al. 2008, 2010), possibly marking the end of the acceleration and collimation zone. Standing shocks playing a major role in the high-energy flaring events have been pinpointed with mm-VLBI also downstream in the jet. In OJ 287, a secondary standing shock at 
a distance of 14 parsecs from the innermost stationary feature was proposed to give rise to a prominent $\gamma$-ray flare as it was crossed by a turbulent moving blob (Agudo et al. 2011). In 3C 345, Schinzel et al. (2012) showed that $\gamma$-rays may be produced at multiple locations over an extended region of 23 parsecs, including the proximity of a stationary feature observed $\sim 10$ parsecs away from the core.

While the physical processes triggering the activity may vary from flare to flare, all the above results point toward a "far dissipation" scenario. Although, as we discussed in Sect. 3, observing at millimeter wavelengths enables us to unveil emission regions closer to the jet base, the millimeter core is still located at parsec distances from the black hole, at least in the brightest blazars (e.g., Sikora et al. 2008; Fromm et al. 2015). The inferred co-spatiality of the low and high-energy emission implies that efficient particle acceleration takes place far beyond the broad-line region. At the distances determined by mm-VLBI studies, the most likely mechanisms giving rise to the high-energy emission are then the synchrotron self-Compton and the external inverse Compton of infra-red photons from the torus.

This conclusion may still be valid when the $\gamma$-ray activity is found to precede the variations in the radio band (see, e.g., Rani et al. 2014; Karamanavis et al. 2016a; Lisakov et al. 2017). For instance, through a dedicated VLBI campaign at 43 and $86 \mathrm{GHz}$ of the high-redshift blazar PKS 1502+106, Karamanavis et al. (2016a) determined that the high-energy emission originates few parsecs upstream of the mm-core (Fig. 6). With the combined effort of single-dish and VLBI studies, an absolute distance of $\sim 1.9 \mathrm{pc}$ was inferred for the location of the $\gamma$-ray activity with respect to the black hole (Karamanavis et al. 2016b). Since the broad line region is expected to extend on much smaller scales in this object ( $\sim 0.1 \mathrm{pc}$, Abdo et al. 2010$)$, an external seed photon field is most likely provided by the infrared torus also in this case.

Next to the described findings, a number of cases which may require more complex interpretations have been reported in the literature. "Orphan" $\gamma$-ray flares, with no structural change on parsec scales, have been frequently observed, e.g., in Mrk 421 (Lico et al. 2014) and CTA 102 (Casadio et al. 2015b). In the misaligned object $3 \mathrm{C} 84$, the variability in the radio and at the very high $\gamma$-ray energies $(E>10 \mathrm{GeV})$ appears to be uncorrelated (Suzuki et al. 2012; Nagai et al. 2012). Particularly for the TeV BL Lacs and for radio galaxies, whose Doppler factors inferred from VLBI kinematic analyses are found to be too low to account for the observed energetic processes, a simple "one-zone model" for the high-energy production may be not appropriate. For these objects, it was suggested (Ghisellini et al. 2005) that the inverse Compton emission can be enhanced if the jet is structured transversely, e.g., it is formed by a fast central spine and a slower outer sheath. Under these conditions, the electrons of one component can up-scatter the beamed photons of the other, so that the low- and the high-energy emissions do not necessarily originate in the same region, and can show uncorrelated variability. A similar mechanism was theorized by Georganopoulos and Kazanas (2003) for decelerating jets, i.e., those characterized by a radial velocity gradient. $43 \mathrm{GHz}$ VLBI observations of $3 \mathrm{C} 84$ have revealed that this jet is in fact characterized by a transverse structure of the spine-sheath kind (Fig. 7, Nagai et al. 2014). The uncorrelated radio- $\gamma$ variability, the observed transverse stratification, and modeling of the SED favor a "two-zone model" for the high-energy production in this source (Tavecchio and Ghisellini 2014). 
B. Boccardi et al.

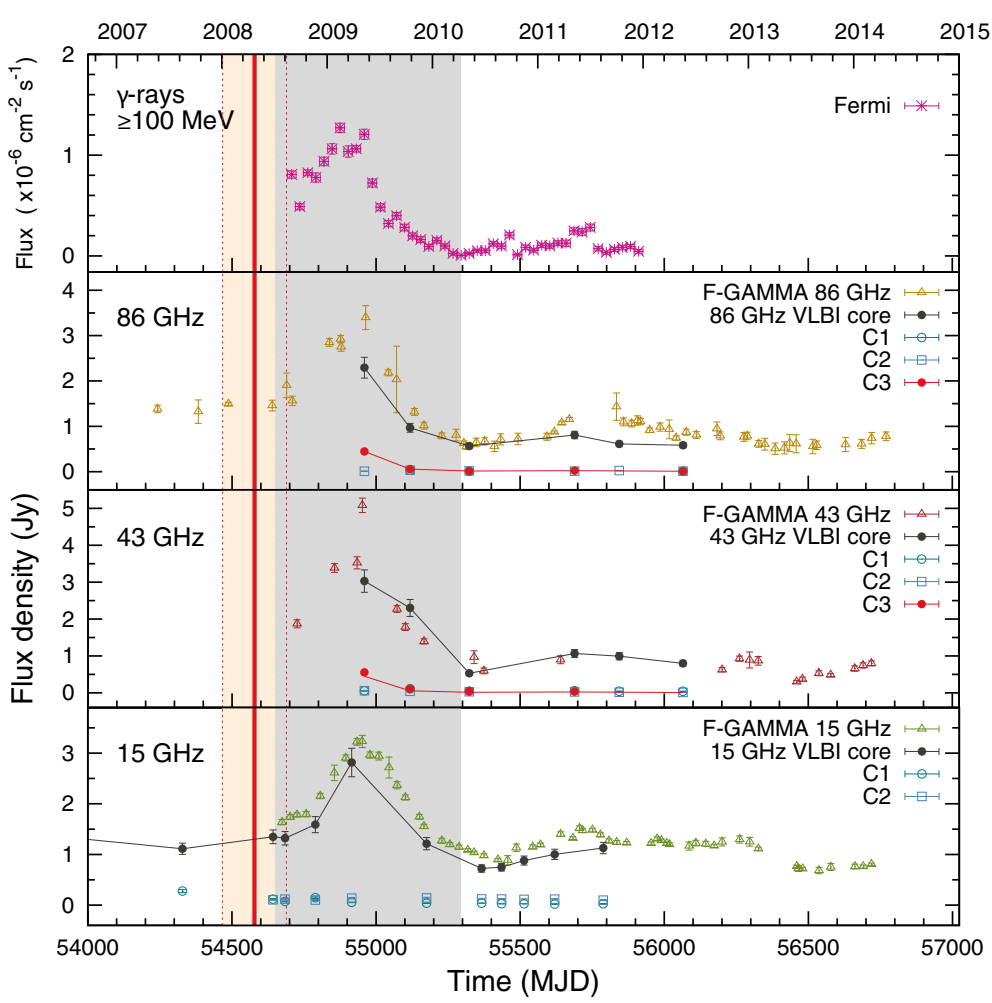

Fig. 6 Light curves of the blazar PKS 1502+106, which showed a very prominent $\gamma$-ray flare in 2009 (Karamanavis et al. 2016a). From top to bottom: (1) monthly binned Fermi/LAT $\gamma$-ray light curve at energies $E>100 \mathrm{MeV}$; (2) F-GAMMA single-dish radio light curve at $86 \mathrm{GHz}$, core and component light curves from the VLBI flux density decomposition; (3) same as above, at $43 \mathrm{GHz}$; (4) same as above, at $15 \mathrm{GHz}$. A newly ejected VLBI knot, labeled C3, is visible at 43 and $86 \mathrm{GHz}$. Its ejection time, designated by the red solid line and estimated based on the VLBI kinematic analysis, follows the onset of the $\gamma$-ray flare. The latter originates $\sim 2$ parsecs away from the black hole, downstream of the broad line region (Image reproduced with permission from Karamanavis et al. 2016b, copyright by ESO)

Transversely stratified jets in BL Lacs have been proposed (Tavecchio et al. 2014) as possible sources of the neutrinos detected by IceCube in the (0.1-1) PeV range (Aartsen et al. 2013). These detections have opened new frontiers for the study of the high-energy emission in AGN, since several recent studies have identified a number of blazars as possible counterparts of the IceCube signals (e.g., Kadler et al. 2016; Padovani et al. 2016). Future mm-VLBI analysis of the structural characteristics of these candidates may provide further clues for the correctness of the proposed associations.

\subsection{Internal structure and morphology}

As evident from the previous section, probing the morphology and the internal structure of the innermost regions of AGN jets is essential for truly understanding the physical 


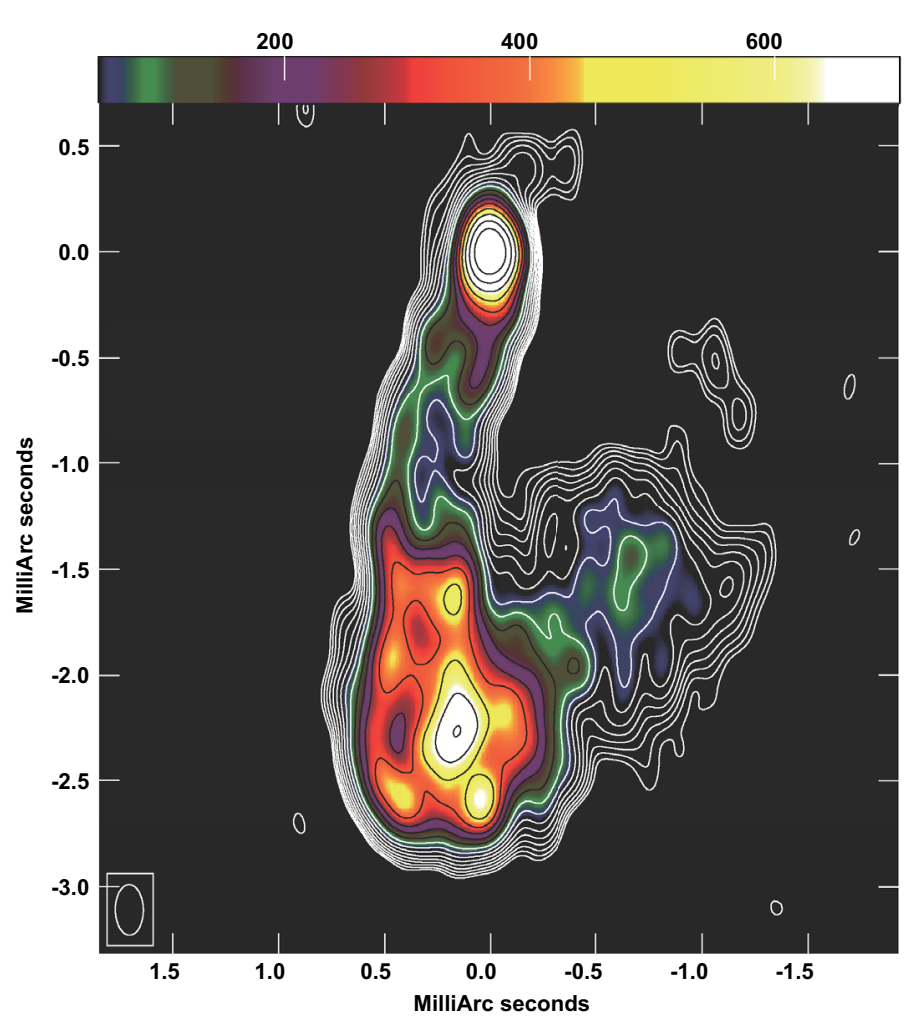

Fig. 7 The limb-brightened jet structure of the peculiar radio galaxy 3C 84 as revealed by VLBI observations at $43 \mathrm{GHz}$ (Image reproduced with permission from Nagai et al. 2014, copyright by AAS)

processes occurring in the plasma flow. Analyzing the jet transverse structure is not only important for the correct modeling of the high-energy emission, as explained, but also for probing the development of plasma instabilities, which can affect crucially the jet propagation on larger scales.

To obtain meaningful insights into the internal jet structure, it is necessary to resolve the flow in the transverse direction. This condition is usually not met in centimeter VLBI observations, especially in the most compact regions at the jet base. Interferometric observations with enhanced resolution, like space or mm-VLBI, are instead adequate for achieving such a goal in several nearby objects.

A powerful tool for testing the development of instabilities is the analysis of the jet ridge line, i.e., of the evolution of the location of the peak of emission in the transverse direction as a function of distance from the core. In the case of the strong jet in S5 0836+710 (Perucho et al. 2012), VLBI observations up to $43 \mathrm{GHz}$ have shown that the ridge line is likely tracing a pattern of helically twisted pressure maxima due to the formation of Kelvin-Helmholtz instabilities. A similar conclusion was reached in the analysis of 3C 273 through space VLBI observations at $5 \mathrm{GHz}$ (Lobanov and Zensus 2001). In this case, a double helical ridge line is observed, the jet being bright at its limbs and dimmer close to the central axis. 


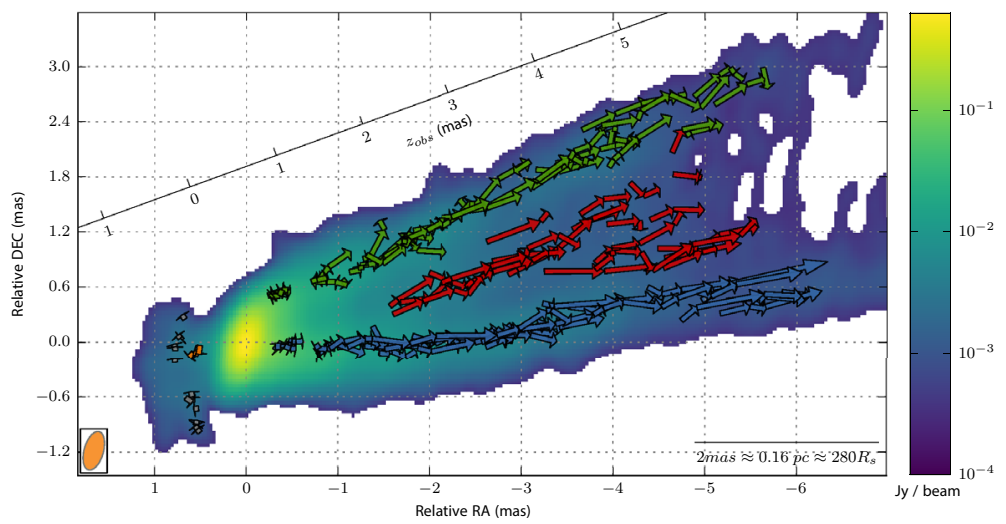

Fig. 8 Velocity structure of the jet in M87 derived from the analysis of a multiepoch VLBI data set at $43 \mathrm{GHz}$. Motion is detected along three main filaments, two outer limbs and a central body. Two overlapping velocity components can be identified: a mildly relativistic one and a faster streamline with a Lorentz factor of 2.5 (Image reproduced with permission from Mertens et al. 2016, copyright by ESO)

The class formed by the so-called "structured jets", i.e., those characterized by a transverse gradient in the intensity, in the velocity, or in the polarization properties (see Sect. 4.3), is getting more and more numerous as the resolution of the images improves. Several limb-brightened jets could recently be imaged through mm-VLBI, e.g., in 3C 84 (Nagai et al. 2014, Fig. 7), in Mrk 501 (Giroletti et al. 2008), in Mrk 421 (Piner et al. 2010), in M 87 (Junor et al. 1999; Walker et al. 2016; Mertens et al. 2016), and in Cygnus A (Boccardi et al. 2016a, b). In the latter source, the stratification is visible not only in the approaching jet, but also in the counter-jet, and a similar feature is hinted in the weak counter-jet of M87. The limb-brightening characterizing these jets could reflect the presence of instability patterns, as for 3C 273, or it may be a direct result of the jet formation mechanism. This second scenario appears especially plausible for M 87, where the double ridge line is visible already at few tens of Schwarzschild radii from the central engine. The intensity gradient can, in this case, be explained in two ways: (1) the jet emissivity is intrinsically lower closer to the jet axis or (2) the jet emissivity is apparently lower close to the jet axis due to the existence of a spinesheath velocity gradient. In fact, limb-brightening will naturally arise in sufficiently misaligned jets due to the differential boosting of each filament or to the de-boosting of the fast central spine. A spine-sheath velocity structure has been long proposed for relativistic jets (Sol et al. 1989), and is compatible with findings from recent kinematic studies in Cygnus A (Boccardi et al. 2016b) and M 87 (Mertens et al. 2016). In particular, Mertens et al. (2016) were able to obtain a detailed 2-D velocity field in the inner regions of M 87, clearly showing the displacement of features along three main filaments of the flow, two outer limbs and a central body (Fig. 8). Two speed components, which appear to overlap across the jet, are detected: a mildly relativistic speed likely associated with an outer disk wind or with an instability pattern and a faster streamline featuring a Lorentz factor of $\sim 2.5$.

These results point out the importance of resolving transversely the plasma flow in order to better understand the fundamental processes involved in its formation, a 
subject which will be more widely debated in the final part of this article. Another morphological aspect which is emerging as a characteristic feature of jets in mm-VLBI images, and that may be also intimately related to the launching mechanism, is the large apparent bending of emission features in the immediate surroundings of the core, also known as "jet wobbling". Changes in the direction of the jet axis appear to be more dramatic close to the jet apex than on the scales probed by cm-VLBI observations. Strongly bending features, which are often stationary, are seen on sub-milliarcsecond scales in objects like Mrk 501 (Koyama et al. 2016), OJ 287 (Hodgson et al. 2017), 3C 279 (Lu et al. 2013), NRAO 150 (Agudo et al. 2007), CTA 102 (Fromm et al. 2013), and other sources (e.g., Rantakyrö et al. 1998). At present, no clear explanation exists for their appearance. In some objects, the wobbling appears to be characterized by a periodicity, as suggested for the blazar $4 C+12.50$ (Lister et al. 2003). In this case, a regular precession of the accretion disk, either induced by a binary black hole system or by the presence of some other massive object located in the nuclear regions, appears as a viable explanation for the periodic change of direction. In most of the cases, however, the jet bending is rather erratic. In OJ 287, Agudo et al. (2012b) exclude the existence of well-defined periodicity of the inner-jet swing (the 12 year periodic precession proposed by Tateyama and Kingham 2004, for larger scales), and rather favor phenomena of asymmetric injection of plasma due to turbulence in the accretion disk or of instabilities in the innermost jet. If the magnetic field is still dynamically important on the scales probed by mm-VLBI, current-driven instabilities (Benford 1981) are expected to manifest. Among them, the kink instability is potentially the most disruptive (McKinney and Blandford 2009; Mignone et al. 2010), and was recently suggested to play a critical role for the origin of the FR I/FR II dichotomy in radio galaxies (Tchekhovskoy and Bromberg 2016).

\subsection{Polarization and magnetic field topology}

Polarimetric studies can be a powerful tool for deriving fundamental constraints on the jet physics, as well as on the properties of the jet environment. The interpretation of polarization data requires, however, particular caution. The likely co-existence of relativistic, projection and other effects makes it challenging to reconstruct the intrinsic orientation and strength of the magnetic fields (see, e.g., Lyutikov et al. 2005). Moreover, the very nature of these fields is still highly debated. In particular, it is unclear whether the observed polarization has to be ascribed to the local ordering, e.g., due to compression in a shock, of an otherwise random ambient magnetic field, or to the presence of a large-scale, ordered field permeating the plasma flow. While a certain degree of ambiguity is likely inevitable when investigating this subject, we discuss here how VLBI observations at millimeter wavelengths enable many of the uncertainties to be reduced.

From synchrotron theory, the predicted fractional linear polarization for an ensemble of relativistic electrons moving in a uniform magnetic field is 70-75\%, in the case of optically thin emission, and 10-12\% for optically thick emission (Pacholczyk 1970). However, both single-dish radio surveys (e.g., Aller et al. 1985, 1992) and VLBI studies of large samples (e.g., Pollack et al. 2003; Lister and Homan 2005; 
Helmboldt et al. 2007) have inferred much lower degrees than expected on this theoretical basis. Typical values measured in the proximity of the VLBI core are below $5 \%$, while there is a trend of increasing fractional polarization, up to tens of percent, at larger distances from the jet base (e.g., Lister and Homan 2005).

These results have often been interpreted as evidence for the random nature of the magnetic field (see Hughes 2005, and references therein). Under the assumption that large part of the jet emission originates in shocked regions, polarization degrees of 20-30\%, or higher, can be explained in the framework of the shock-in-jet model (Marscher and Gear 1985; Hughes et al. 1985), as a consequence of the compression and amplification of the component of the magnetic field oriented parallel to the shock front. In the case of a transverse shock, the electric vector position angle (EVPA) is then expected to be oriented in the direction parallel to the jet axis, a configuration which is frequently found to characterize the compact jet knots in polarimetric VLBI images, especially in BL Lac objects (Gabuzda et al. 2000). Enhanced polarization can also result from the formation of oblique and conical shocks (Cawthorne and Cobb 1990, and references therein) or from the shear of the magnetic field lines at the boundaries between the jet and the ambient medium (Laing 1980).

Although a largely random field can be adequate for reproducing the observed properties of AGN jets, its assumption poses some difficulties for theoretical models describing the launching, acceleration, and collimation of the flow. In fact, most of these models require the existence of an ordered, large scale field at the base of the jet (e.g., Spruit 2010, and references therein). If this prediction is correct, the aforementioned observational results can then be explained in two ways. A first possibility is that the field gets disrupted and tangled, e.g., owing to magnetic instabilities (Giannios and Spruit 2006), already before the jet becomes visible at the VLBI core. This scenario is plausible especially in the case of the most highly boosted blazars, where, as we said, the mm-VLBI core appears to be located quite far from the central engine, at a distance of $10^{4}-10^{5}$ Schwarzschild radii (e.g., Marscher et al. 2008; Fromm et al. 2015). Alternatively, a large-scale field may be preserved on VLBI scales, and the radio emission may be intrinsically highly polarized, but a strong depolarization occurs as the radiation propagates to the observer. In general, depolarization results as a consequence of the integration of polarized emission from multiple emission regions and/or along the line of sight. In relativistic jets, a decrease of the net polarization could be observed in the following cases:

\section{- Line of sight integration through opaque material}

Assuming that the VLBI core actually marks, at a given frequency, the transition region to the optically thin regime, then at least part of its emission is expected to be opaque. The line of sight integration through a partially optically thick material could be responsible for the low net polarization observed at the core location. This effect may apply especially to sources which are seen at a very small viewing angles.

\section{- Beam depolarization}

VLBI observations are characterized by a finite resolution. If the observing beam is not negligible when compared to the size of the coherent polarization structure 
of the source, depolarization may result from the averaging of polarized emission inside the interferometer beam.

\section{- Faraday depolarization}

In addition to the previous effects, further depolarization could arise as a consequence of Faraday rotation (Burn 1966), i.e., the rotation of the polarization plane of an electromagnetic wave while it propagates through a magnetized plasma. The rotation measure $R M$ quantifies, at a given wavelength $\lambda$, the difference between the intrinsic polarization angle $\chi_{0}$ and the observed one $\chi$. In the simplest case of a homogeneous medium, $R M$ is proportional to the integral over the line of sight from the source to the observer of the density of charges in the medium $n_{e}$ times the component of the magnetic field along the line of sight $B_{\|}$:

$$
\chi=R M \lambda^{2}+\chi_{0}, \quad R M \propto \int n_{e} B_{\|} \mathrm{d} l
$$

The presence of Faraday screens can cause a reduction of the total polarization whenever the emission experiences differential rotations while propagating to the observer. This can happen either when the Faraday rotating material is mixed with the emitting plasma (internal depolarization) or when the radiation crosses external inhomogeneous media (external depolarization). It is important to note that the strongest rotations are expected to be induced by thermal electrons, whose presence is likely abundant in the jet environment. Dense thermal media which can potentially act as Faraday screens include both orbiting clouds in the broad- or narrow-line region and non-relativistic material embedding the relativistic jet beam (disk winds). Multi-frequency cm-VLBI studies have indeed inferred the presence of such media from the high rotation measures (up to thousands of $\mathrm{rad} / \mathrm{m}^{2}$ ) detected in several blazars (e.g., Taylor 1998; Zavala and Taylor 2004; Hovatta et al. 2012. The effect appears to be especially strong at shorter distances from the central engine, indicating a decreasing strength of the magnetic field with distance and/or a decreasing density of the thermal electrons. The latter is in agreement with the expectations from the unified scheme for AGN that most of the dense gas surrounding the accretion disk is concentrated in the central parsecs.

Ultimately, while a large-scale field may still permeate the plasma flow on small VLBI scales, these very scales are also the most heavily obscured, synchrotron self-absorbed and unresolved. VLBI at millimeter wavelengths is then the optimal technique when aiming at revealing the intrinsic strength and orientation of the magnetic fields in the jet. Due to its dependence on the square of the wavelength (Eq. 7), Faraday rotation is expected to be diminished, and so is the depolarization arising from it. Moreover, being able to penetrate the jet base closer to the central engine, mm-VLBI increases the possibility to image-with enhanced resolution-those regions potentially characterized by dynamically important magnetic fields.

A clear example of the potential of mm-VLBI observations is provided, in this context, by the study of BL Lac presented by Marscher et al. (2008). At $43 \mathrm{GHz}$, polarized emission could be detected from a region upstream of the VLBI core (Fig. 9). The disturbance injected at the jet base propagates downstream, causing a double flare 


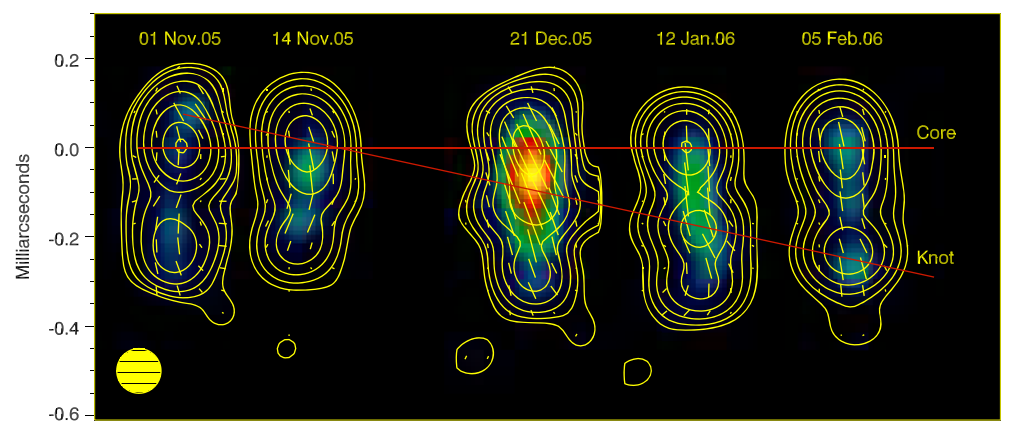

Fig. 9 VLBI polarimetric images of BL Lac at $43 \mathrm{GHz}$. A feature emerging in the region upstream of the core is visible in the first three maps. The optical and radio polarization properties suggest this region to be part of the jet acceleration and collimation zone (Image reproduced with permission from Marscher et al. 2008, copyright by NPG)

at optical, $\mathrm{X}$-rays and $\mathrm{TeV}$ energies. The second flare is also observed in radio when the disturbance crosses the VLBI core, which is interpreted as a standing shock. The optical and radio polarization properties suggest the upstream region to be part of the acceleration and collimation zone of this jet. In particular, the smooth rotation of the optical polarization vector by $\sim 240^{\circ}$ observed over few days and its final alignment with the radio polarization vector, parallel to the jet axis, support the presence of a large scale magnetic field with helical geometry which then becomes mostly turbulent close to the standing shock. In this scenario, consistent with the findings from RadioAstron at $22 \mathrm{GHz}$ (Gómez et al. 2016) and similarly suggested for PKS 1510-089 (Marscher et al. 2010), the VLBI core is the location where the plasma has attained its terminal speed, and possibly equipartition of the magnetic and particle energy densities.

In the picture proposed by Marscher et al. (2008), the helical structure of the magnetic field is already disrupted at the mm-VLBI core, located at a distance of $\sim 10^{4} R_{S}$ from the jet apex. Several other authors, however, have suggested that the helical geometry may be preserved on much larger scales. The existence of a parsec-scale helical field has been proposed for NRAO 150 (Molina et al. 2014) for explaining the high speed rotation of the emission regions. In the polarimetric analysis of this object performed up to $86 \mathrm{GHz}$ frequencies, a toroidal magnetic field is observed, although the polarization degree is quite low due to the almost perfectly face-on orientation of this jet. In Zamaninasab et al. (2013), the nature of a broad arc-like feature in the inner regions of 3C 345.3 was investigated through multi-frequency, polarimetric VLBI imaging between 5 and $86 \mathrm{GHz}$. Transverse gradients across the arc, which is highly polarized, are observed both in the polarization degree and in the apparent magnetic vector position angle, which varies by more than $90^{\circ}$. Moreover, the rotation measure shows a sign reversal across the feature. These properties can be best reproduced by assuming the presence of a large-scale helical field. If confirmed, this result has the remarkable implication that an ordered field exists at distance of $\sim 10^{7} R_{\mathrm{S}}$, i.e., much beyond the expected extension of the acceleration and collimation region. Transverse rotation measure gradients have been observed in several other objects on parsec scales (e.g., Asada et al. 2002; Gabuzda et al. 2004; O’Sullivan and Gabuzda 
2009), and often interpreted as a characteristic signature of helical fields. The analysis of RM transverse gradients can provide important insights for the investigation of this subject. However, as pointed out by Taylor and Zavala (2010), spurious results can arise as a consequence of limited transverse resolution in the VLBI images. Therefore, it will be important in the future to verify the aforementioned results through VLBI studies at the highest possible resolution.

Polarimetric imaging at frequencies higher than $43 \mathrm{GHz}$ has been limited in the past due to the non-standard calibration procedures required. Over the past few years, a pipeline for the calibration of full-polarization, global VLBI data at $86 \mathrm{GHz}$ has been developed, and the feasibility of the technique has been demonstrated for the case of the blazar 3C 345 (Martí-Vidal et al. 2012). Recently, a polarimetric image of M 87 has also been produced at this frequency (Hada et al. 2016), revealing the presence of a highly polarized feature in the innermost regions of the jet. Concerning the earlier experiments, $86 \mathrm{GHz}$ polarization maps had been obtained for mainly three objects: 3C 120 (Gómez et al. 1999), 3C 273 (Attridge 2001; Attridge et al. 2005), and 3C 279 (Attridge 2001). No polarization was detected for the cores of 3C 120 and 3C273, while a weakly polarized core was observed in $3 \mathrm{C} 379$. At larger distances from the central engine, instead, highly polarized features emerge in all the three cases, with the magnetic field oriented parallel to the jet axis in 3C 120 and 3C 273, and perpendicular in $3 \mathrm{C} 279$. If the absence of polarization in the core is due to Faraday depolarization only, extremely high rotation measures, larger than $90000 \mathrm{rad} \mathrm{m}^{-2}$ in the case of 3C 273, are inferred. While, on the one hand, several alternative explanations exist for the low polarization of VLBI cores, such high rotation measures are not incompatible with the tremendous opacities that can be expected at the jet base. Through recent ALMA observations of the high-redshift blazar PKS 1330-211 at frequencies up to $300 \mathrm{GHz}$ ( $1 \mathrm{THz}$ in the source frame), rotation measures of the order or $\sim 10^{8} \mathrm{rad}$ $\mathrm{m}^{-2}$ have been derived (Martí-Vidal et al. 2015). These values are consistent with the presence of dynamically important magnetic fields at the jet base, with strengths of tens of Gauss and potentially much higher. The importance of this and other similar results is further commented in the following section.

\subsection{Launching, acceleration and collimation}

VLBI studies at centimeter wavelengths, as well as radio interferometric analyses probing larger scales, have collected substantial observational evidence of one basic fact: jets are self-similar over many orders of magnitude in length. This self-similarity is expected to break close to the black hole, where the jet should be dominated by the magnetic energy driving it. Among the proposed mechanisms for jet formation, many of which involve a purely hydrodynamical launching (Blandford and Rees 1974), the magnetic launching model (see, e.g., Meier et al. 2001) appears as the most viable option according to numerical simulations (e.g., Tchekhovskoy 2015, and references therein), and is currently largely favored. In this model, the jet properties scale trivially with the black hole mass, conveniently explaining the similarities between galactic and extragalactic jets. 
Although there is a general agreement on the basic principles of the magnetic launching mechanism, reproducing the extreme properties observed in relativistic jets, such as opening angles smaller than $1^{\circ}$ and bulk Lorentz factors as high as 50 , is still among the most challenging tasks for theorists. This is firstly due to the inherent complexity of plasma physics in the immediate surrounding of super-massive black holes, whose description requires a full GRMHD (General Relativistic MagnetoHydroDynamic) formalism. In the past 20 years, important steps forward have been made by implementing three-dimensional GRMHD numerical simulations (Koide et al. 1999; McKinney 2006). These have shown that AGN jets can be efficiently powered by the rotational energy of the compact central object, extracted through strong, large scale magnetic fields. The power source can either be the accretion disk, as described in the work of (Blandford and Payne 1982), or a spinning super-massive black hole, as originally proposed by Blandford and Znajek (1977).

At their very base, such magnetically driven jets are likely to propagate in the form of pure Poynting flux, which then gets gradually converted into kinetic flux and, for a small percentage, into radiation. Based on theoretical models (Vlahakis and Königl 2004; Lyubarsky 2009) and numerical simulations (McKinney 2006; Komissarov et al. 2007), the predicted scales for the mechanisms of acceleration and collimation to take place span an interval between few and $10^{3}-10^{5}$ Schwarzschild radii, corresponding to sub-parsec or parsec scales (see Fig. 5). In this region, the magnetic field is expected to be dynamically important and well ordered, with a geometry gradually evolving from purely poloidal to helical, or possibly to purely toroidal.

In the recent years, mm-VLBI observations have been able to probe these previously unexplored scales following different approaches. In addition to the analysis of the polarization properties, discussed in the previous section, important observational tests could be provided either through statistical studies in survey experiments or through a direct and detailed imaging of single, optimal targets.

The most recent surveys conducted at $86 \mathrm{GHz}$ (Lobanov et al. 2000; Lee et al. 2008; Gopalakrishnan Nair et al. 2016) enabled to model the observed distribution of brightness temperature, and to investigate its dependence as a function of distance from the central engine. A significant trend has emerged, indicating that the brightness temperature measured at the location of the $86 \mathrm{GHz}$ core, peaking at $T_{\mathrm{b}} \sim 10^{11} \mathrm{~K}$ (Lee et al. 2008), is lower than the one measured at lower frequencies. This behavior can be best explained by assuming that plasma acceleration is still taking place in the region between the VLBI cores (Lee et al. 2016), a result which confirms the theoretical prediction that the acceleration zone extends on parsec scales. These findings, together with many of the previously discussed polarization and variability studies (e.g., Marscher et al. 2008, 2010), collocate the mm-core in a crucial position: in blazars the mm-core marks the transition region between the magnetic-dominated and the kinetic-dominated regimes.

One implication of this scenario is that, in most sources, the bulk of the MHD processes driving the outflow may occur in the "invisible" jet, comprised between the supermassive black hole and the mm-core. There exists, however, a limited sample of objects where a high-resolution imaging of the true jet base can be obtained and MHD models for jet formation can be tested in detail. The list of suitable targets mainly includes nearby misaligned jets (Table 2) where, for reasons which are not fully 
Table 2 List of nearby, misaligned objects which are well suited for mm-VLBI observations aimed at imaging the jet formation region

\begin{tabular}{llll}
\hline Source & $D_{\mathrm{L}}[\mathrm{Mpc}]$ & $\log \left(\mathrm{M}_{\mathrm{BH}}\left[\mathrm{M}_{\odot}\right]\right)$ & Linear size of $50 \mu$ as $\left[R_{\mathrm{S}}\right]$ \\
\hline M87 & 19 & $9.8^{\mathrm{a}}$ & 8 \\
Cygnus A & 252 & $9.4^{\mathrm{b}}$ & 229 \\
3C 84 & 77 & $8.9^{\mathrm{c}}$ & 235 \\
NGC 1052 & 22 & $8.2^{\mathrm{d}}$ & 354 \\
Centaurus A & 8 & $7.7^{\mathrm{e}}$ & 397 \\
\hline
\end{tabular}

Col. 1: Source name. Col. 2: Luminosity distance (assuming a $\Lambda \mathrm{CDM}$ cosmology with $H_{0}=$ $\left.69.6 \mathrm{~h}^{-1} \mathrm{~km} \mathrm{~s}^{-1} \mathrm{Mpc}^{-1}, \Omega_{\mathrm{M}}=0.286, \Omega_{\Lambda}=0.714\right) D_{\mathrm{L}}$. Col. 3: Logarithm of the black hole mass $\mathrm{M}_{\mathrm{BH}}$, given in units of solar masses $\mathrm{M}_{\odot}$. Ref: ${ }^{\mathrm{a}}$ Gebhardt et al. (2011), ${ }^{\mathrm{b}}$ Tadhunter et al. (2003), ${ }^{\mathrm{c}}$ Scharwächter et al. (2013), ${ }^{\mathrm{d}}$ Woo and Urry (2002), ${ }^{\mathrm{e}}$ Neumayer (2010). Col. 4: Linear size in units of Schwarzschild radii probed by VLBI at $86 \mathrm{GHz}$. The objects are listed in order of decreasing spatial resolution

clarified (Marscher 2011) but are most likely related to the reduced impact of projection and relativistic effects, the VLBI core appears to be located much closer to the central black hole than in blazars. For instance, phase-referencing VLBI observations of M 87 (Hada et al. 2011) have determined a shift of only 14-23 $R_{\mathrm{S}}$ of the $43 \mathrm{GHz}$ core with respect to the central engine, while an upper limit of $100 R_{\mathrm{S}}$ could be inferred for NGC 1052 at $86 \mathrm{GHz}$ (Baczko et al. 2016). Table 2 indicates that the resolution provided by VLBI at $86 \mathrm{GHz}$ is sufficient for probing the acceleration and collimation region in all of the listed objects, while the mechanisms involved in the jet launching, expected to take place on scales $<100 R_{\mathrm{S}}$ (Sect. 3), can be currently best studied in M 87. The latter is the optimal target in terms of spatial resolution, although the intermediate orientation of its jet $\left(\theta \sim 17^{\circ}\right.$, Mertens et al. 2016) introduces larger uncertainties on the intrinsic parameters than in true radio galaxies like Cygnus A $\left(\theta \sim 75^{\circ}\right.$, Boccardi et al. 2016b) or NGC 1052 (64 $<\theta<87^{\circ}$, Baczko et al. 2016).

In M87, the jet formation region has been investigated in numerous studies. The collimation zone is well resolved already at $43 \mathrm{GHz}$; on sub-parsec scales, the limbbrightened flow appears much broader than on parsec scales, expanding with an opening angle of $\sim 60^{\circ}$ (Junor et al. 1999; Ly et al. 2004). Deeper imaging at $86 \mathrm{GHz}$ (Hada et al. 2016) reveals an even broader jet base with apparent opening angle of $\sim 100^{\circ}$, indicating that the jet starts poorly collimated and gets rapidly focused farther downstream. These findings are confirmed by the analysis of a more recent $86 \mathrm{GHz}$ image, obtained after stacking five different maps (Fig. 10, Kim et al. 2016). The stacking method is especially effective when aiming at recovering the full jet cross-section and at investigating with better fidelity the jet expansion. Both studies performed at $86 \mathrm{GHz}$ show that the jet has a parabolic shape on scales of $\sim 100 \mathrm{R}_{S}$. Using a rich, multi- frequency VLBI data set, Asada and Nakamura (2012) inferred that this shape is preserved also on larger scales, up to approximately $\sim 10^{5} \mathrm{R}_{S}$. Beyond that, however, the flow appears to be freely expanding, i.e., it has a conical shape. According to theory, acceleration through magnetohydrodynamic processes is inefficient in conical flows, and can extend on large scales only if the plasma undergoes differential collimation (see, e.g., Komissarov 2012). The observed transition from a parabolic 


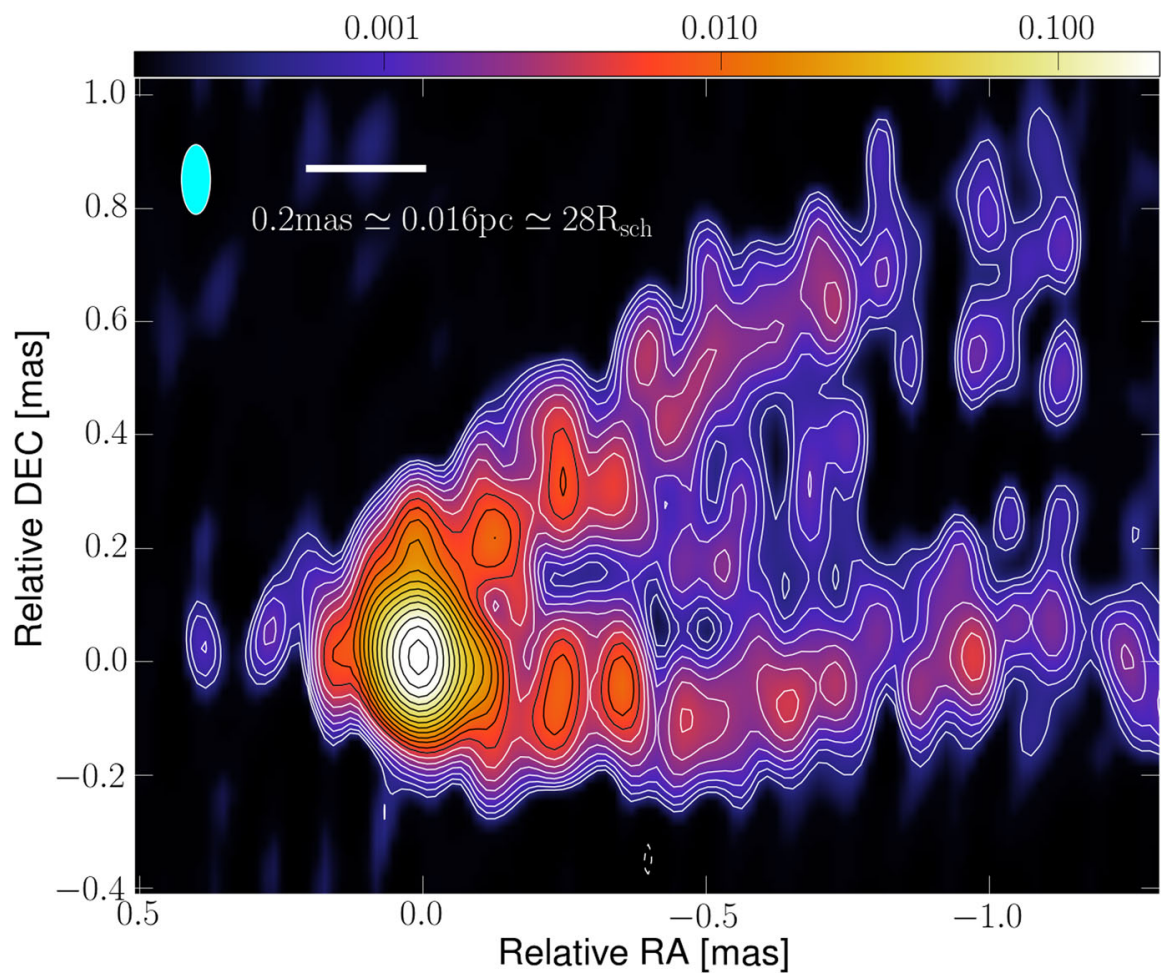

Fig. 10 VLBI observations of M 87 at $86 \mathrm{GHz}$ (Image reproduced with permission from Kim et al. 2016, copyright by the authors). The image was created after stacking 5 VLBI images taken during 2004-2015

to a conical shape may then signal a crucial change in the physical conditions of the plasma, marking the termination of the acceleration zone. Interestingly, the transition occurs in the vicinity of the Bondi radius (where a change in the ambient pressure gradient is expected) and of a stationary feature in the HST-1 complex, a bright region where superluminal speeds of $4 c-6 c$ have been measured (Biretta et al. 1999). Then, a natural question to ask is: does the flow show acceleration in the parabolic region comprised between its base and HST-1? Previous kinematic studies (Biretta et al. 1995; Kovalev et al. 2007; Ly et al. 2007; Asada et al. 2014) have yielded contrasting results, likely due to the poor sampling and to the intrinsic difficulty in identifying moving features in a jet that lacks well defined spots, and is transversely stratified. The method recently employed by Mertens et al. (2016) enabled a detailed two-dimensional kinematic structure to be inferred and, therefore, appears as the most appropriate in the case of M 87 (Fig. 8). In this work, accelerating features are detected on scales from $10^{2}$ to $10^{4} R_{\mathrm{S}}$; the speeds, which are mildly relativistic, increase faster up to distances of $10^{3} R_{\mathrm{S}}$, while a milder gradient is observed further downstream. These properties could be well interpreted in the framework of MHD acceleration and collimation: the bulk of the acceleration occurs within thousands of Schwarzschild radii, and is followed by a regime of saturation of the Poynting flux conversion which may extend up to HST-1. 


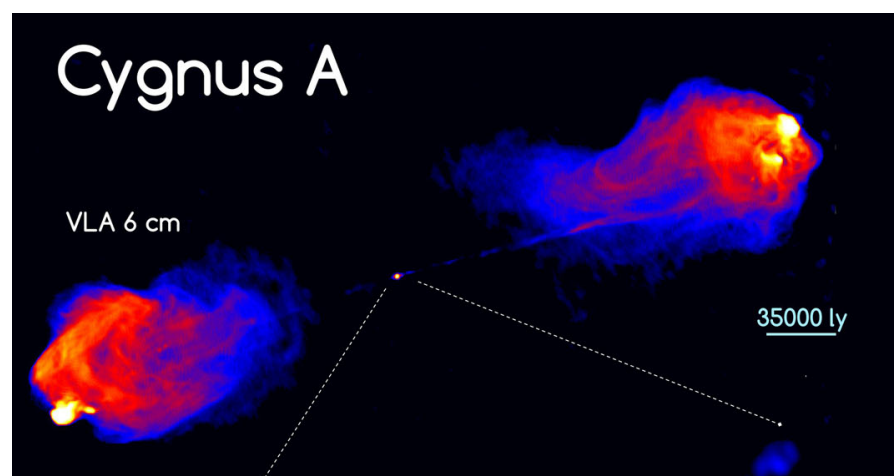

VLBI $6 \mathrm{~cm}$

30 ly

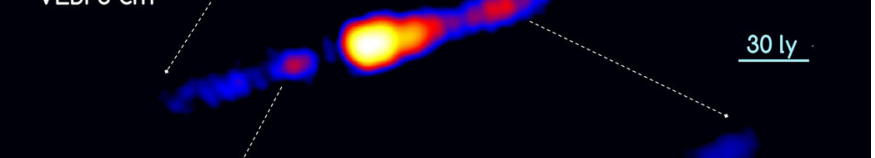

VLBI $2 \mathrm{~cm}$

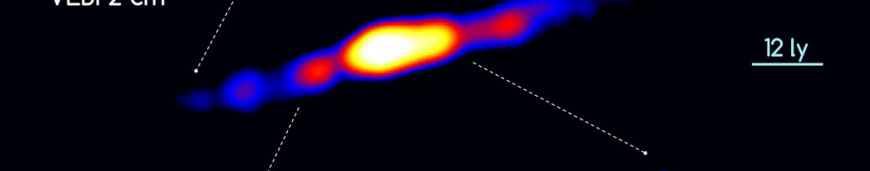

VLBI $7 \mathrm{~mm}$

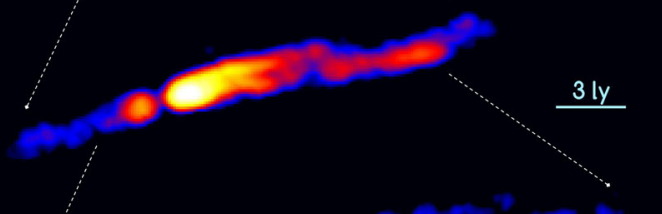

VLBI $3 \mathrm{~mm}$

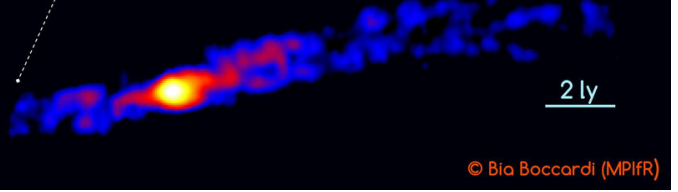

Fig. 11 The radio galaxy Cygnus A on scales from hundreds of kilo-parsecs imaged with the Very Large Array (Perley et al. 1984) to the sub-parsec probed with mm-VLBI (Boccardi et al. 2016a, b). The VLBI images are created after stacking several epochs. Data at $2 \mathrm{~cm}$ are from the MOJAVE survey (Lister et al. 2009)

The acceleration and collimation region could also be imaged in Cygnus $\mathrm{A}$ at 43 and $86 \mathrm{GHz}$ (Boccardi et al. 2016a,b). A multi-scale representation of this prototypical source is shown in Fig. 11. The observed kinematic properties in this powerful radio galaxy are quite similar to those inferred for M 87: the flow is transversely stratified and mildly relativistic $(1<\Gamma<2.5)$, it has a parabolic shape up to scales of $10^{3}-10^{4} R_{S}$, and shows acceleration with two distinct regimes, a fast regime (with Lorentz factor increasing as fast as the jet radius) up to $\sim 5000 R_{S}$ and a slow regime up to $\sim 10^{4} R_{S}$. At $86 \mathrm{GHz}$, the two-sided jet could be resolved transversely also close to the apex, revealing a very wide jet base with a minimum width of (227 \pm 98$) R_{\mathrm{S}}$. This value is much larger than the radius of the innermost stable circular orbit (ISCO) 
in the accretion disk $\left(1<R_{\mathrm{ISCO}}<9 R_{\mathrm{S}}\right)$, implying that at least part of the flow must be anchored at large disk radii in the accretion disk.

In this respect, Cygnus A differs from M 87. Pilot observations of M 87 performed at $1 \mathrm{~mm}$ with the Event Horizon Telescope (EHT) (Doeleman et al. 2012) could determine an upper limit for the transverse size of the jet apex of only $5.5 R_{\mathrm{S}}$, compatible with later findings by Krichbaum et al. (2014). Unlike in Cygnus A, the initial jet width in M 87 is comparable with the radius of the innermost stable circular orbit (ISCO), suggesting that the jet is launched from the inner regions of the disk or from the ergosphere. Very similar conclusions were reached by Mertens et al. (2016) through the analysis of the jet rotation at $43 \mathrm{GHz}$.

All the aforementioned results agree well with the theoretical predictions for magnetically dominated jets, supporting the currently most favored paradigm. Additional observational grounds favoring the magnetic launching scenario come from estimates of the magnetic field strength at the jet apex. In a recent analysis of a large number of sources from the MOJAVE survey (Zamaninasab et al. 2014), the nuclear magnetic field strength has been derived from the jet radio luminosity. The inferred forces are extremely high, and comparable with the black hole's gravitational pull. Magnetic field strengths of the order of $\sim 10^{3} \mathrm{G}$ or more were also derived in GMVA studies of OJ 287 (Hodgson et al. 2017) and NGC 1052 (Baczko et al. 2016), and may be compatible with the already discussed ALMA results presented by Martí-Vidal et al. (2015) for PKS 1330-211. The existence of the so-called Magnetically Arrested Disks (MADs), i.e., disks featuring dynamically important magnetic fields, was theorized by Narayan et al. (2003). The adjective "arrested" refers to the accretion, which can be suppressed if the magnetic pressure gets strong enough, in the innermost regions of the disk.

Numerical simulations (Tchekhovskoy et al. 2011) confirm that MAD systems can produce strong jets, with powers up to $140 \%$ of the accreted rest-mass energy in the case of a maximally spinning black hole. Simply speaking, this means that "more than what comes in goes out", and this surplus must be at the expenses of the black hole's rotational energy. This theoretical prediction agrees very well with another recent observational study of a large sample of blazars (Ghisellini et al. 2014), showing that the jet luminosity is correlated with the disk luminosity but at the same time is much larger than it. Again, the contribution of a spinning black hole is necessary to account for the jet power. These results indicate that the Blandford-Znajeck process is appropriate for reproducing the properties of relativistic jets in blazars. If, on the other hand, the Blandford-Payne mechanism is also at play, but produces less powerful jets, it is natural to expect that the black-hole driven component will be dominant due to the stronger boosting. The observation of disk winds in highly misaligned sources like Cygnus A is well placed in such a scenario.

A deep investigation of other objects in Table 2 is ongoing. In the future, the possibility of enlarging the sample of sources where the jet formation region can be imaged will depend on the compactness of the jet bases at frequencies higher than $86 \mathrm{GHz}$. Results from the surveys (Lobanov et al. 2000; Lee et al. 2008; Gopalakrishnan Nair et al. 2016) indicate a decrease of brightness temperature with increasing frequency, which may hinder the detection of the innermost jet emission at $230 \mathrm{GHz}$ and higher. On the other hand, experiments planned for the near future will also profit from the 
deployment of the phased ALMA and from the improved sensitivity of global arrays, which may enable to probe the jet formation region in fainter objects.

\subsection{Sagittarius A*}

Sagittarius A* $\left(\operatorname{Sgr} A^{*}\right)$ is one of the three main components forming the Sagittarius A complex, located at the center of the Milky way. This compact object, emitting from radio to $\gamma$-rays with a peak at sub-millimeter wavelengths, is believed to mark the position of the supermassive black hole at the center of our galaxy. With a mass of about 4 millions solar masses (Gillessen et al. 2009) and a distance of $\sim 8.3$ kiloparsecs (Reid et al. 2014), Sgr A* is characterized by the largest apparent event horizon size of any black hole candidate in the Universe, which makes it a privileged cosmic laboratory for studies aimed at resolving the innermost accretion flow on Schwarzschild radius scales. The latter is not only important for understanding the accretion mechanisms and the formation of possible outflows, but also for verifying fundamental physics. On such scales, strong gravity effects are expected to be at play, and crucial tests of general relativity may be performed (Johannsen et al. 2016a, b).

In the radio band, the galactic center is characterized by a nearly flat radio spectrum with a flux density of approximately $1 \mathrm{Jy}$. The source is compact and well detected on VLBI scales. Since the first single-baseline detection at $215 \mathrm{GHz}$ in 1995 (Krichbaum et al. 1997, 1998), several test observations of Sgr A* have been conducted in this band. While successful VLBI experiments have been performed also at 43 and $86 \mathrm{GHz}$ (e.g., Krichbaum et al. 1993; Lu et al. 2011; Rauch et al. 2016; Ortiz-León et al. 2016), observing at even higher frequencies is essential in this object in order to partially overcome the strong broadening caused from interstellar scattering. This affects quite heavily the emission at 43 and $86 \mathrm{GHz}$, and is still of the order of $20 \mu$ as at $215 \mathrm{GHz}$ (Bower et al. 2006). In a three-station VLBI experiment at the latter frequency, a scattering-corrected source size of $37 \mu$ as could be determined (Doeleman et al. 2008). Assuming the aforementioned distance and mass parameters, the radius of the black hole event horizon subtends an angular scales of $\sim 10 \mu \mathrm{as}$, but the apparent horizon is expected to be enlarged due to gravitational lensing, to a diameter of $\sim 52 \mu$ as. Therefore, quite surprisingly, the size measured at $215 \mathrm{GHz}$ was found to be smaller than the apparent dimension of the event horizon. This remarkable result suggests that the emission from $\operatorname{Sgr}^{*}$ may not be centered on the exact location of the black hole but it may originate in its surroundings.

Several models have been elaborated with the aim of identifying the nature of the emission region. Many invoke the emission from an inefficient accretion flow, such as the ADAF (advection-dominated accretion flow, Narayan and Yi 1994) and the ADIOS (advection-dominated inflow-outflow solutions, Blandford and Begelman 1999). While these models may explain the observed emission up to the high energies, they have been found to significantly underpredict the low-frequency radio continuum (e.g., Yuan et al. 2002). The latter component can be better reproduced by including the contribution of hot electrons $\left(T \sim 10^{11} \mathrm{~K}\right)$, often proposed to originate in a weak relativistic jet coupled to the accretion flow (e.g., Falcke and Markoff 2000). With a bolometric luminosity several orders of magnitude below the Eddington limit 


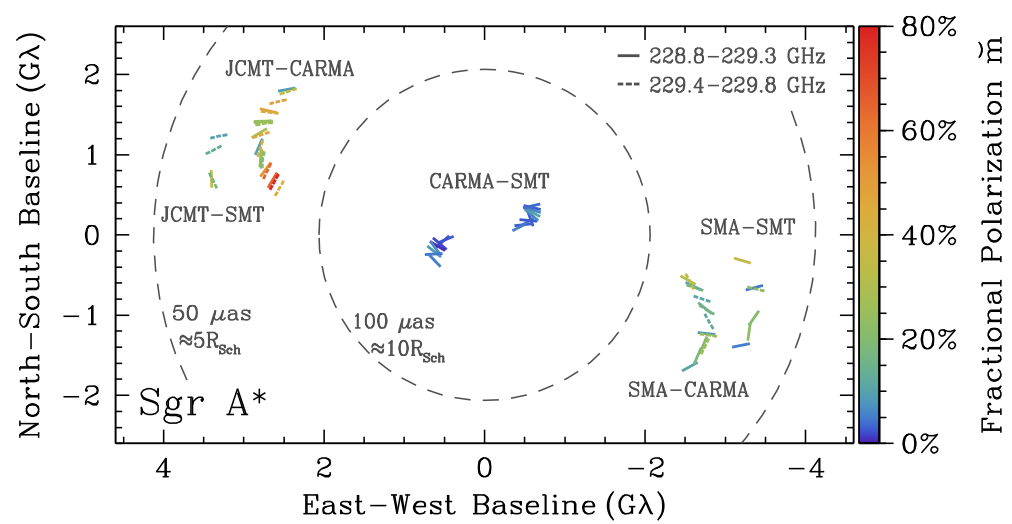

Fig. 12 Interferometric fractional polarization measurements for Sgr A* during EHT observations in 2013. Polarization degrees up to 70-80\% are observed. The telescopes participating to the experiment were the phased CARMA (Combined Array for Research in Millimeter-wave Astronomy) in California, the SMT (Submillimeter Telescope) in Arizona, the SMA (Submillimeter Array) and the JCMT (James Clerk Maxwell Telescope) in Hawaii (Image reproduced with permission from Johnson et al. 2015, copyright by AAAS)

( $\sim 10^{-8} L_{\text {Edd }}$ ), Sgr $A *$ is highly under-luminous when compared to a classical AGN. The study of this putative jet is, therefore, fundamental for exploring the jet formation phenomenon under different physical conditions.

In the near future, the Event Horizon Telescope will be capable of producing detailed imaging of the radio structure in Sgr A*, possibly shedding light on the nature of the emission. In the meantime, the visibility and closure phase data collected so far at $230 \mathrm{GHz}$ are already providing important constraints. The radio emission has been observed to vary on time scales of days (Fish et al. 2011), and a persistent asymmetry in the source structure has been deduced from the non-zero closure phases (Fish et al. 2016b). Moreover, a recent study has shown that the radio emission is highly polarized, up to $\sim 70 \%$, on scales of $6 \mathrm{R}_{S}$ (Johnson et al. 2015, Fig. 12), and that the polarization direction varies spatially. The polarization degree decreases by one order of magnitude when looking at the short baselines, to values that match very well those measured at the same frequency on arcsecond scales (e.g., Bower et al. 2003). This comparison shows, on the one hand, that the polarization arises in the very central regions of the source, and is not associated with dust emission in the surroundings. Secondly, it points out the absolute importance of resolving these compact regions for unveiling the coherent polarization structures in the source. There is no single explanation for the existence of strong ordered magnetic fields in Sgr A*. They could signal the presence of a magnetically dominated region formed after accumulation of magnetic flux near the event horizon, similarly to that inferred for the cores of extragalactic jets (Sect. 4.4), or they could arise as a consequence of instabilities (e.g., the magnetorotational instability-MRI, Balbus and Hawley 1991) in the magnetized and rotating accretion disk. Finally, they could also be associated with the emergence of a relativistic jet. 
The second and most crucial objective of the EHT project will be the direct imaging of the so-called "black hole shadow". The shadow is the dark region around a black hole (Bardeen 1973; Luminet 1979; Falcke et al. 2000), expected to be observable in the presence of a background source, e.g., an optically thin plasma. The shadow should be surrounded by a thin photon ring, whose shape and size are independent on the physical detail of the accretion process and should only be determined by the basic properties of the black hole, i.e., its mass and spin, and by its inclination. The photon ring is predicted to be perfectly circular in the case of a non-spinning black hole with spherical symmetry, while crescent-like morphologies should arise in the case of a Kerr black hole. Concerning the brightness distribution around the photon ring, this is expected to be asymmetric in all cases due to differential Doppler beaming, but its detailed characteristics will also depend on the physical conditions of the accretion flow (see, e.g., Mościbrodzka et al. 2009; Dexter et al. 2010). Ultimately, the analysis of the geometrical and physical properties of the shadow will provide further constraints on the properties of the plasma flow in SgrA* and, most importantly, a direct test for the validity of the no-hair theorem and of the Einstein's theory of gravity in general.

Through a recent four-site experiment at $230 \mathrm{GHz}$, providing a resolution of $\sim 2.5$ Schwarzschild radii, Lu et al. (in preparation) were able to resolve the event horizon scale structure of $\mathrm{Sgr} \mathrm{A}^{*}$, which revealed a complex brightness distribution compatible with the expectations for a crescent-like structure. VLBI observations in the near future should allow more detailed imaging. Upcoming experiments will offer new challenges to observers, due to the sparse $(u, v)$-coverage, influence of scattering in the images, and time variability during the observations. Several methods are being developed, concerning non-imaging analysis and addressing the mitigation of those effects (Fish et al. 2014; Johnson and Gwinn 2015; Johnson 2016; Lu et al. 2016).

The investigation of the Galactic center is considered the main scientific goal of the Event Horizon Telescope (EHT) project, whose efforts are shared with-and complemented by - the BlackHoleCam European collaboration (e.g., Goddi et al. 2017). In this context, vibrant research activity is being carried out at present on multiple fronts, from the refinement of theoretical models and simulations to the enhancement of VLBI arrays and data analysis techniques. For a recent overview of the EHT efforts concerning these subjects, the reader is referred to Fish et al. (2016a).

\section{The future of mm-VLBI}

Fifty years after its birth, VLBI is experiencing a second youth due to its development in the millimeter and sub-millimeter regimes. Unexplored frontiers have now become accessible thanks to it, new science has been produced and more fundamental results are foreseen in the coming years.

The GMVA is conducting successful biannual observations at $86 \mathrm{GHz}$, and has provided important constraints for theories describing blazar physics and jet formation. In the future, a more reliable description of the jet kinematic properties and variability could be obtained through a high-cadence monitoring which, given the short timescales characterizing blazars, is currently too sparse. Significant improvements could also come by implementing multi-frequency receiving systems, following the 
example given by the Korean VLBI Network (Asada et al. 2017). Besides the obvious advantages it would provide for the accurate characterization of the jet spectral properties, this method also enables to enhance the data quality. By applying a technique known as source-frequency phase referencing, the measurements obtained at the lowest frequencies, which are less affected by atmospheric opacity, can in fact be used to correct the phase at higher frequencies.

In addition to the Korean VLBI Network, which is at present regularly participating in the observing sessions, other telescopes may enhance the future imaging capabilities at $86 \mathrm{GHz}$. These include the NOEMA (NOrthern Extended Millimeter Array), successor of the Plateau du Bure observatory, the Large Millimeter Telescope in Mexico, the phased ALMA (at present commissioned for observing at 86 and $230 \mathrm{GHz}$, potentially capable of going down to $43 \mathrm{GHz}$ and up to $345 \mathrm{GHz}$ and even beyond), and the Greenland Telescope, starting its commissioning in early 2017. These latter sites are also part of the Event Horizon Telescope, together with APEX in Chile, the JCMT (James Clerk Maxwell Telescope) and the SMA (Sub-Millimeter Array), both located in Hawaii, the Arizona Radio Observatory (SMT), the SPT (South Pole Telescope), and the $30 \mathrm{~m}$ telescope atop Pico Veleta in Spain.

The ALMA Phasing Project (e.g., Matthews et al. 2014), led by the Haystack Observatory of the Massachusetts Institute of Technology in collaboration with several institutions worldwide, started in 2011 and completed commissioning in 2015. Its goal was to phase-up the 64 ALMA dishes to form a single VLBI station with equivalent diameter of 84 meters. Additionally to the phasing, the project includes polarization conversion to match the typical circular polarization recorded by the VLBI stations with the X/Y (linear) polarization used by ALMA (Martí-Vidal et al. 2016).

ALMA has performed its first regular VLBI observations in April 2017 during Cycle 4. The GMVA was assigned the role of the network provider at $86 \mathrm{GHz}$, and the Event Horizon Telescope consortium (supervised by the National Radio Astronomy Observatory) this role at $230 \mathrm{GHz}$. Thanks to its large collecting surface, ALMA is expected to provide a substantial boost in sensitivity, since the root-mean-square noise of the image will be typically reduced by a factor of three. The first observations including ALMA are still limited by technical reasons, such as a flux density threshold of $500 \mathrm{mJy}$ and the need of long integration time for polarization calibration. Those limitations will be loosen in the future, for instance, when a sky model can be introduced to the phasing.

The possibility of achieving an excellent sensitivity will be crucial in future EHT experiments, aiming at producing a direct image of the black-hole shadow. High sensitivity and good image fidelity will be obtained not only through the expansion of the array, which after 2020 should also include a telescope in Namibia, Africa (AMTAfrica Millimeter Telescope), ${ }^{9}$ but also thanks to the improvement of the recording capabilities at each site, with subsequent increase in bandwidth. Recording capabilities are now boosted by the use of digital techniques and, from the $512 \mathrm{Mbit} / \mathrm{s}$ used several years ago, present developments in digital base band conversion (e.g., Tuccari et al. 2012) bring them to $64 \mathrm{Gbit} / \mathrm{s}$, yielding instantaneous bandwidths of $16 \mathrm{GHz}$.

\footnotetext{
${ }^{9}$ http://www.ru.nl/rbhh-eng/.
} 
Millimeter VLBI is an example of cutting edge technology at the service of science. The international effort recently spent in its further upgrade will enable the exciting scientific questions presented in this article to be fully addressed, by zooming into some of the most violent phenomena in the Universe. Landmark tests for our current knowledge of fundamental physics will be provided, together with final and direct evidence for the existence of black holes at the center of galaxies.

Acknowledgements Open access funding provided by Max Planck Society. The authors would like to thank the anonymous referee and the editors for their suggestions, which significantly improved the article. The authors are also thankful to Andrei Lobanov, Rebecca Azulay, Carolina Casadio, Vassilis Karamanavis, Ioannis Myserlis, Alice Pasetto, and Thalia Traianou for reading and commenting on the manuscript, and to Uwe Bach for kindly providing the calibrated $6 \mathrm{~cm}$ data of Cygnus A shown in Fig. 11. E.R. was partially supported by the Spanish MINECO grant AYA2015-63939-C2-2-P and by the Generalitat Valenciana grant PROMETEOII/2014/057. This study makes use of $43 \mathrm{GHz}$ VLBA data from the VLBABU Blazar Monitoring Program (VLBA-BU-BLAZAR; http://www.bu.edu/blazars/VLBAproject.html), funded by NASA through the Fermi Guest Investigator Program. The VLBA is an instrument of the Long Baseline Observatory. The Long Baseline Observatory is a facility of the National Science Foundation operated by Associated Universities, Inc. This research has made use of data from the MOJAVE database that is maintained by the MOJAVE team (Lister et al. 2009). The European VLBI Network is a joint facility of European, Chinese, South African, and other radio astronomy institutes funded by their national research councils.

Open Access This article is distributed under the terms of the Creative Commons Attribution 4.0 International License (http://creativecommons.org/licenses/by/4.0/), which permits unrestricted use, distribution, and reproduction in any medium, provided you give appropriate credit to the original author(s) and the source, provide a link to the Creative Commons license, and indicate if changes were made.

\section{References}

Aartsen MG, Abbasi R, Abdou Y, Ackermann M, Adams J, Aguilar JA, Ahlers M, Altmann D, Auffenberg J, Bai X et al (2013) First observation of PeV-energy neutrinos with IceCube. Phys Rev Lett 111(2):021103

Abdo AA, Ackermann M, Ajello M, Atwood WB, Axelsson M, Baldini L, Ballet J, Barbiellini G, Bastieri D, Baughman BM et al (2010) PKS 1502+106: a new and distant gamma-ray blazar in outburst discovered by the Fermi Large Area Telescope. Astrophys J 710:810-827

Acero F, Ackermann M, Ajello M, Albert A, Atwood WB, Axelsson M, Baldini L, Ballet J, Barbiellini G, Bastieri D, Belfiore A, Bellazzini R, Bissaldi E, Blandford RD, Bloom ED, Bogart JR, Bonino R, Bottacini E, Bregeon J, Britto RJ, Bruel P, Buehler R, Burnett TH, Buson S, Caliandro GA, Cameron RA, Caputo R, Caragiulo M, Caraveo PA, Casandjian JM, Cavazzuti E, Charles E, Chaves RCG, Chekhtman A, Cheung CC, Chiang J, Chiaro G, Ciprini S, Claus R, Cohen-Tanugi J, Cominsky LR, Conrad J, Cutini S, D’Ammando F, de Angelis A, DeKlotz M, de Palma F, Desiante R, Digel SW, Di Venere L, Drell PS, Dubois R, Dumora D, Favuzzi C, Fegan SJ, Ferrara EC, Finke J, Franckowiak A, Fukazawa Y, Funk S, Fusco P, Gargano F, Gasparrini D, Giebels B, Giglietto N, Giommi P, Giordano F, Giroletti M, Glanzman T, Godfrey G, Grenier IA, Grondin M-H, Grove JE, Guillemot L, Guiriec S, Hadasch D, Harding AK, Hays E, Hewitt JW, Hill AB, Horan D, Iafrate G, Jogler T, Jóhannesson G, Johnson RP, Johnson AS, Johnson TJ, Johnson WN, Kamae T, Kataoka J, Katsuta J, Kuss M, La Mura G, Landriu D, Larsson S, Latronico L, Lemoine-Goumard M, Li J, Li L, Longo F, Loparco F, Lott B, Lovellette MN, Lubrano P, Madejski GM, Massaro F, Mayer M, Mazziotta MN, McEnery JE, Michelson PF, Mirabal N, Mizuno T, Moiseev AA, Mongelli M, Monzani ME, Morselli A, Moskalenko IV, Murgia S, Nuss E, Ohno M, Ohsugi T, Omodei N, Orienti M, Orlando E, Ormes JF, Paneque D, Panetta JH, Perkins JS, Pesce-Rollins M, Piron F, Pivato G, Porter TA, Racusin JL, Rando R, Razzano M, Razzaque S, Reimer A, Reimer O, Reposeur T, Rochester LS, Romani RW, Salvetti D, Sánchez-Conde M, Saz Parkinson PM, Schulz A, Siskind EJ, Smith DA, Spada F, Spandre G, Spinelli P, Stephens TE, Strong AW, Suson DJ, Takahashi H, Takahashi T, Tanaka Y, Thayer JG, 
Thayer JB, Thompson DJ, Tibaldo L, Tibolla O, Torres DF, Torresi E, Tosti G, Troja E, Van Klaveren B, Vianello G, Winer BL, Wood KS, Wood M, Zimmer S, Fermi-LAT Collaboration (2015) Fermi Large Area Telescope third source catalog. Astrophys J Suppl 218:23

Ackermann M, Ajello M, Allafort A, Angelakis E, Axelsson M, Baldini L, Ballet J, Barbiellini G, Bastieri D, Bellazzini R, Berenji B, Blandford RD, Bloom ED, Bonamente E, Borgland AW, Bouvier A, Bregeon J, Brez A, Brigida M, Bruel P, Buehler R, Buson S, Caliandro GA, Cameron RA, Cannon A, Caraveo PA, Casandjian JM, Cavazzuti E, Cecchi C, Charles E, Chekhtman A, Cheung CC, Ciprini S, Claus R, Cohen-Tanugi J, Cutini S, de Palma F, Dermer CD, EdCe Silva, Drell PS, Dubois R, Dumora D, Escande L, Favuzzi C, Fegan SJ, Focke WB, Fortin P, Frailis M, Fuhrmann L, Fukazawa Y, Fusco P, Gargano F, Gasparrini D, Gehrels N, Giglietto N, Giommi P, Giordano F, Giroletti M, Glanzman T, Godfrey G, Grandi P, Grenier IA, Guiriec S, Hadasch D, Hayashida M, Hays E, Healey SE, Jóhannesson G, Johnson AS, Kamae T, Katagiri H, Kataoka J, Knödlseder J, Kuss M, Lande J, Lee S-H, Longo F, Loparco F, Lott B, Lovellette MN, Lubrano P, Makeev A, Max-Moerbeck W, Mazziotta MN, McEnery JE, Mehault J, Michelson PF, Mizuno T, Monte C, Monzani ME, Morselli A, Moskalenko IV, Murgia S, Naumann-Godo M, Nishino S, Nolan PL, Norris JP, Nuss E, Ohsugi T, Okumura A, Omodei N, Orlando E, Ormes JF, Ozaki M, Paneque D, Pavlidou V, Pelassa V, Pepe M, Pesce-Rollins M, Pierbattista M, Piron F, Porter TA, Rainò S, Razzano M, Readhead A, Reimer A, Reimer O, Richards JL, Romani RW, Sadrozinski HF-W, Scargle JD, Sgrò C, Siskind EJ, Smith PD, Spandre G, Spinelli P, Strickman MS, Suson DJ, Takahashi H, Tanaka T, Taylor GB, Thayer JG, Thayer JB, Thompson DJ, Torres DF, Tosti G, Tramacere A, Troja E, Vandenbroucke J, Vianello G, Vitale V, Waite AP, Wang P, Winer BL, Wood KS, Yang Z, Ziegler M (2011) The radio/gamma-ray connection in active galactic nuclei in the era of the Fermi Large Area Telescope. Astrophys J 741:30

Ackermann M, Anantua R, Asano K, Baldini L, Barbiellini G, Bastieri D, Becerra Gonzalez J, Bellazzini R, Bissaldi E, Blandford RD, Bloom ED, Bonino R, Bottacini E, Bruel P, Buehler R, Caliandro GA, Cameron RA, Caragiulo M, Caraveo PA, Cavazzuti E, Cecchi C, Cheung CC, Chiang J, Chiaro G, Ciprini S, Cohen-Tanugi J, Costanza F, Cutini S, D’Ammando F, de Palma F, Desiante R, Digel SW, Di Lalla N, Di Mauro M, Di Venere L, Drell PS, Favuzzi C, Fegan SJ, Ferrara EC, Fukazawa Y, Funk S, Fusco P, Gargano F, Gasparrini D, Giglietto N, Giordano F, Giroletti M, Grenier IA, Guillemot L, Guiriec S, Hayashida M, Hays E, Horan D, Jóhannesson G, Kensei S, Kocevski D, Kuss M, La Mura G, Larsson S, Latronico L, Li J, Longo F, Loparco F, Lott B, Lovellette MN, Lubrano P, Madejski GM, Magill JD, Maldera S, Manfreda A, Mayer M, Mazziotta MN, Michelson PF, Mirabal N, Mizuno T, Monzani ME, Morselli A, Moskalenko IV, Nalewajko K, Negro M, Nuss E, Ohsugi T, Orlando E, Paneque D, Perkins JS, Pesce-Rollins M, Piron F, Pivato G, Porter TA, Principe G, Rando R, Razzano M, Razzaque S, Reimer A, Scargle JD, Sgrò C, Sikora M, Simone D, Siskind EJ, Spada F, Spinelli P, Stawarz L, Thayer JB, Thompson DJ, Torres DF, Troja E, Uchiyama Y, Yuan Y, Zimmer S (2016) Minute-timescale $>100 \mathrm{MeV} \gamma$-ray variability during the giant outburst of quasar 3C 279 observed by Fermi-LAT in 2015 June. Astrophys J 824:L20

Agudo I, Bach U, Krichbaum TP, Marscher AP, Gonidakis I, Diamond PJ, Perucho M, Alef W, Graham DA, Witzel A, Zensus JA, Bremer M, Acosta-Pulido JA, Barrena R (2007) Superluminal non-ballistic jet swing in the quasar NRAO 150 revealed by mm-VLBI. Astron Astrophys 476:L17-L20

Agudo I, Jorstad SG, Marscher AP, Larionov VM, Gómez JL, Lähteenmäki A, Gurwell M, Smith PS, Wiesemeyer H, Thum C, Heidt J, Blinov DA, D'Arcangelo FD, Hagen-Thorn VA, Morozova DA, Nieppola E, Roca-Sogorb M, Schmidt GD, Taylor B, Tornikoski M, Troitsky IS (2011) Location of $\gamma$-ray flare emission in the jet of the BL Lacertae object OJ287 more than $14 \mathrm{pc}$ from the central engine. Astrophys J 726:L13

Agudo I, Gómez JL, Casadio C, Cawthorne TV, Roca-Sogorb M (2012a) A recollimation shock 80 mas from the core in the jet of the radio galaxy 3C 120: observational evidence and modeling. Astrophys J 752:92

Agudo I, Marscher AP, Jorstad SG, Gómez JL, Perucho M, Piner BG, Rioja M, Dodson R (2012b) Erratic jet wobbling in the BL Lacertae object OJ287 revealed by sixteen years of $7 \mathrm{~mm}$ VLBA observations. Astrophys J 747:63

Aharonian FA (2000) TeV gamma rays from BL Lac objects due to synchrotron radiation of extremely high energy protons. New Astron 5:377-395

Aharonian F, Akhperjanian AG, Bazer-Bachi AR, Behera B, Beilicke M, Benbow W, Berge D, Bernlöhr K, Boisson C, Bolz O, Borrel V, Boutelier T, Braun I, Brion E, Brown AM, Bühler R, Büsching I, Bulik T, Carrigan S, Chadwick PM, Clapson AC, Chounet L-M, Coignet G, Cornils R, Costamante L, Degrange B, Dickinson HJ, Djannati-Ataï A, Domainko W, Drury LO, Dubus G, Dyks J, Egberts K, 
Emmanoulopoulos D, Espigat P, Farnier C, Feinstein F, Fiasson A, Förster A, Fontaine G, Funk S, Funk S, Füßling M, Gallant YA, Giebels B, Glicenstein JF, Glück B, Goret P, Hadjichristidis C, Hauser D, Hauser M, Heinzelmann G, Henri G, Hermann G, Hinton JA, Hoffmann A, Hofmann W, Holleran M, Hoppe S, Horns D, Jacholkowska A, de Jager OC, Kendziorra E, Kerschhaggl M, Khélifi B, Komin N, Kosack K, Lamanna G, Latham IJ, Le Gallou R, Lemière A, Lemoine-Goumard M, Lenain J-P, Lohse T, Martin JM, Martineau-Huynh O, Marcowith A, Masterson C, Maurin G, McComb TJL, Moderski R, Moulin E, de Naurois M, Nedbal D, Nolan SJ, Olive J-P, Orford KJ, Osborne JL, Ostrowski M, Panter M, Pedaletti G, Pelletier G, Petrucci P-O, Pita S, Pühlhofer G, Punch M, Ranchon S, Raubenheimer BC, Raue M, Rayner SM, Renaud M, Ripken J, Rob L, Rolland L, Rosier-Lees S, Rowell G, Rudak B, Ruppel J, Sahakian V, Santangelo A, Saugé L, Schlenker S, Schlickeiser R, Schröder R, Schwanke U, Schwarzburg S, Schwemmer S, Shalchi A, Sol H, Spangler D, Stawarz Ł, Steenkamp R, Stegmann C, Superina G, Tam PH, Tavernet J-P, Terrier R, van Eldik C, Vasileiadis G, Venter C, Vialle JP, Vincent P, Vivier M, Völk HJ, Volpe F, Wagner SJ, Ward M, Zdziarski AA (2007) An exceptional very high energy gamma-ray flare of PKS 2155-304. Astrophys J 664:L71-L74

Aleksić J, Ansoldi S, Antonelli LA, Antoranz P, Babic A, Bangale P, Barres de Almeida U, Barrio JA, Becerra González J, Bednarek W et al (2014) MAGIC gamma-ray and multi-frequency observations of flat spectrum radio quasar PKS 1510-089 in early 2012. Astron Astrophys 569:A46

Aller HD, Aller MF, Latimer GE, Hodge PE (1985) Spectra and linear polarizations of extragalactic variable sources at centimeter wavelengths. Astrophys J Suppl 59:513-768

Aller MF, Aller HD, Hughes PA (1992) Pearson-Readhead survey sources-properties of the centimeterwavelength flux and polarization of a complete radio sample. Astrophys J 399:16-28

Arshakian TG, León-Tavares J, Lobanov AP, Chavushyan VH, Shapovalova AI, Burenkov AN, Zensus JA (2010) Observational evidence for the link between the variable optical continuum and the subparsecscale jet of the radio galaxy 3C 390.3. Mon Not R Astron Soc 401:1231-1239

Asada K, Nakamura M (2012) The Structure of the M87 jet: a transition from parabolic to conical streamlines. Astrophys J 745:L28

Asada K, Inoue M, Uchida Y, Kameno S, Fujisawa K, Iguchi S, Mutoh M (2002) A helical magnetic field in the jet of 3C 273. Publ Astron Soc Japan 54:L39-L43

Asada K, Nakamura M, Doi A, Nagai H, Inoue M (2014) Discovery of sub- to superluminal motions in the M87 jet: an implication of acceleration from sub-relativistic to relativistic speeds. Astrophys J 781:L2

Asada K, Kino M, Honma M, Hirota T, Lu R-S, Inoue M, Sohn B-W, Shen Z-Q, Ho PTP, Akiyama K, Algaba J, An T, Bower G, Byun D, Dodson R, Doi A, Edwards PG, Fujisawa K, Gu M, Hada K, Hagiwara Y, Jaroenjittichai P, Jung T, Kawashima T, Koyama S, Lee S, Matsushita S, Nagai H, Nakamura M, Niinuma K, Phillips C, Park J, Pu H, Ro H, Stevens J, Trippe S, Wajima K, Zhao G (2017) White paper on East Asian vision for mm/submm VLBI: toward black hole astrophysics down to angular resolution of $1 \sim \mathrm{R}_{S}$. arXiv: 1705.04776

Attridge JM (2001) $86 \mathrm{GHz}$ very long baseline polarimetry of 3C 273 and 3C 279 with the coordinated millimeter VLBI array. Astrophys J 553:L31-L34

Attridge JM, Wardle JFC, Homan DC (2005) Concurrent 43 and $86 \mathrm{GHz}$ very long baseline polarimetry of 3C 273. Astrophys J 633:L85-L88

Atwood WB, Abdo AA, Ackermann M, Althouse W, Anderson B, Axelsson M, Baldini L, Ballet J, Band DL, Barbiellini G et al (2009) The large area telescope on the Fermi gamma-ray space telescope mission. Astrophys J 697:1071-1102

Baczko A-K, Schulz R, Kadler M, Ros E, Perucho M, Krichbaum TP, Böck M, Bremer M, Grossberger C, Lindqvist M, Lobanov AP, Mannheim K, Martí-Vidal I, Müller C, Wilms J, Zensus JA (2016) A highly magnetized twin-jet base pinpoints a supermassive black hole. Astron Astrophys 593:A47

Balbus SA, Hawley JF (1991) A powerful local shear instability in weakly magnetized disks. I-Linear analysis. II-Nonlinear evolution. Astrophys J 376:214-233

Bardeen JM (1973) Timelike and null geodesics in the Kerr metric. In: Dewitt C, Dewitt BS (eds) Black holes (Les Astres Occlus), pp 215-239

Barthel PD (1989) Is every quasar beamed? Astrophys J 336:606-611

Beasley AJ, Dhawan V, Doeleman S, Phillips RB (1997) CMVA observations of compact AGNs. In: Barvainis R, Phillips RB (eds) Millimeter-VLBI science workshop, p 53

Bell AR (1978) The acceleration of cosmic rays in shock fronts. I. Mon Not R Astron Soc 182:147-156

Belloni T, (ed) (2010) The jet paradigm. Lecture notes in physics, vol 794. Springer, Berlin

Benford G (1981) Stability of galactic radio jets. Astrophys J 247:792-802

Biretta JA, Zhou F, Owen FN (1995) Detection of proper motions in the M87 jet. Astrophys J 447:582 
Biretta JA, Sparks WB, Macchetto F (1999) Hubble space telescope observations of superluminal motion in the M87 jet. Astrophys J 520:621-626

Blake GM (1972) Fluid dynamic stability of double radio sources. Mon Not R Astron Soc 156:67

Blandford RD, Begelman MC (1999) On the fate of gas accreting at a low rate on to a black hole. Mon Not R Astron Soc 303:L1-L5

Blandford RD, Königl A (1979) Relativistic jets as compact radio sources. Astrophys J 232:34-48

Blandford RD, Payne DG (1982) Hydromagnetic flows from accretion discs and the production of radio jets. Mon Not R Astron Soc 199:883-903

Blandford RD, Pringle JE (1976) Kelvin-Helmholtz instability of relativistic beams. Mon Not R Astron Soc 176:443-454

Blandford RD, Rees MJ (1974) A 'twin-exhaust' model for double radio sources. Mon Not R Astron Soc 169:395-415

Blandford RD, Znajek RL (1977) Electromagnetic extraction of energy from Kerr black holes. Mon Not R Astron Soc 179:433-456

Błażejowski M, Sikora M, Moderski R, Madejski GM (2000) Comptonization of infrared radiation from hot dust by relativistic jets in quasars. Astrophys J 545:107-116

Boccardi B, Krichbaum TP, Bach U, Bremer M, Zensus JA (2016a) First 3 mm-VLBI imaging of the two-sided jet in Cygnus A. Zooming into the launching region. Astron Astrophys 588:L9

Boccardi B, Krichbaum TP, Bach U, Mertens F, Ros E, Alef W, Zensus JA (2016b) The stratified two-sided jet of Cygnus A. Acceleration and collimation. Astron Astrophys 585:A33

Bower GC, Wright MCH, Falcke H, Backer DC (2003) Interferometric detection of linear polarization from Sagittarius A* at $230 \mathrm{GHz}$. Astrophys J 588:331-337

Bower GC, Goss WM, Falcke H, Backer DC, Lithwick Y (2006) The intrinsic size of Sagittarius A* from 0.35 to $6 \mathrm{~cm}$. Astrophys J 648:L127-L130

Burn BJ (1966) On the depolarization of discrete radio sources by Faraday dispersion. Mon Not RAS 133:67

Casadio C, Gómez JL, Grandi P, Jorstad SG, Marscher AP, Lister ML, Kovalev YY, Savolainen T, Pushkarev $\mathrm{AB}$ (2015a) The connection between the radio jet and the gamma-ray emission in the radio galaxy $3 \mathrm{C}$ 120. Astrophys J 808:162

Casadio C, Gómez JL, Jorstad SG, Marscher AP, Larionov VM, Smith PS, Gurwell MA, Lähteenmäki A, Agudo I, Molina SN, Bala V, Joshi M, Taylor B, Williamson KE, Arkharov AA, Blinov DA, Borman GA, Di Paola A, Grishina TS, Hagen-Thorn VA, Itoh R, Kopatskaya EN, Larionova EG, Larionova LV, Morozova DA, Rastorgueva-Foi E, Sergeev SG, Tornikoski M, Troitsky IS, Thum C, Wiesemeyer $\mathrm{H}$ (2015b) A multi-wavelength polarimetric study of the blazar CTA 102 during a gamma-ray flare in 2012. Astrophys J 813:51

Cawthorne TV, Cobb WK (1990) Linear polarization of radiation from oblique and conical shocks. Astrophys J 350:536-544

Celotti A, Fabian AC (1993) The kinetic power and luminosity of parsecscale radio jets—an argument for heavy jets. Mon Not R Astron Soc 264:228

Celotti A, Ghisellini G, Chiaberge M (2001) Large-scale jets in active galactic nuclei: multiwavelength mapping. Mon Not R Astron Soc 321:L1-L5

Cohen MH, Meier DL, Arshakian TG, Homan DC, Hovatta T, Kovalev YY, Lister ML, Pushkarev AB, Richards JL, Savolainen T (2014) Studies of the jet in B1 Lacertae. I. Recollimation shock and moving emission features. Astrophys J 787:151

Daly RA, Marscher AP (1988) The gasdynamics of compact relativistic jets. Astrophys J 334:539-551

D’Arcangelo FD, Marscher AP, Jorstad SG, Smith PS, Larionov VM, Hagen-Thorn VA, Kopatskaya EN, Williams GG, Gear WK (2007) Rapid multiwaveband polarization variability in the quasar PKS 0420-014: optical emission from the compact radio jet. Astrophys J 659:L107-L110

Dermer CD, Schlickeiser R (1993) Model for the high-energy emission from blazars. Astrophys J 416:458

Dexter J, Agol E, Fragile PC, McKinney JC (2010) The submillimeter bump in Sgr A* from relativistic MHD simulations. Astrophys J 717:1092-1104

Doeleman SS, Weintroub J, Rogers AEE, Plambeck R, Freund R, Tilanus RPJ, Friberg P, Ziurys LM, Moran JM, Corey B, Young KH, Smythe DL, Titus M, Marrone DP, Cappallo RJ, Bock DC-J, Bower GC, Chamberlin R, Davis GR, Krichbaum TP, Lamb J, Maness H, Niell AE, Roy A, Strittmatter P, Werthimer D, Whitney AR, Woody D (2008) Event-horizon-scale structure in the supermassive black hole candidate at the Galactic Centre. Nature 455:78-80

Doeleman SS, Fish VL, Schenck DE, Beaudoin C, Blundell R, Bower GC, Broderick AE, Chamberlin R, Freund R, Friberg P, Gurwell MA, Ho PTP, Honma M, Inoue M, Krichbaum TP, Lamb J, Loeb A, 
Lonsdale C, Marrone DP, Moran JM, Oyama T, Plambeck R, Primiani RA, Rogers AEE, Smythe DL, SooHoo J, Strittmatter P, Tilanus RPJ, Titus M, Weintroub J, Wright M, Young KH, Ziurys LM (2012) Jet-launching structure resolved near the supermassive black hole in M87. Science 338:355

Falcke H, Markoff S (2000) The jet model for Sgr A*: radio and X-ray spectrum. Astron Astrophys 362:113118

Falcke H, Melia F, Agol E (2000) Viewing the shadow of the black hole at the galactic center. Astrophys J 528:L13-L16

Falcke H, Körding E, Markoff S (2004) A scheme to unify low-power accreting black holes. Jet-dominated accretion flows and the radio/X-ray correlation. Astron Astrophys 414:895-903

Fanaroff BL, Riley JM (1974) The morphology of extragalactic radio sources of high and low luminosity. Mon Not R Astron Soc 167:31P-36P

Ferrari A, Trussoni E, Zaninetti L (1978) Relativistic Kelvin-Helmholtz instabilities in extragalactic radiosources. Astron Astrophys 64:43-52

Fish VL, Doeleman SS, Beaudoin C, Blundell R, Bolin DE, Bower GC, Chamberlin R, Freund R, Friberg P, Gurwell MA, Honma M, Inoue M, Krichbaum TP, Lamb J, Marrone DP, Moran JM, Oyama T, Plambeck R, Primiani R, Rogers AEE, Smythe DL, SooHoo J, Strittmatter P, Tilanus RPJ, Titus M, Weintroub J, Wright M, Woody D, Young KH, Ziurys LM (2011) $1.3 \mathrm{~mm}$ Wavelength VLBI of Sagittarius A*: detection of time-variable emission on event horizon scales. Astrophys J 727:L36

Fish VL, Johnson MD, Lu R-S, Doeleman SS, Bouman KL, Zoran D, Freeman WT, Psaltis D, Narayan R, Pankratius V, Broderick AE, Gwinn CR, Vertatschitsch LE (2014) Imaging an event horizon: mitigation of scattering toward Sagittarius A*. Astrophys J 795:134

Fish V, Akiyama K, Bouman K, Chael A, Johnson M, Doeleman S, Blackburn L, Wardle J, Freeman W (2016a) Observing — and imaging — active galactic nuclei with the Event Horizon Telescope. Galaxies $4: 54$

Fish VL, Johnson MD, Doeleman SS, Broderick AE, Psaltis D, Lu R-S, Akiyama K, Alef W, Algaba JC, Asada K, Beaudoin C, Bertarini A, Blackburn L, Blundell R, Bower GC, Brinkerink C, Cappallo R, Chael AA, Chamberlin R, Chan C-K, Crew GB, Dexter J, Dexter M, Dzib SA, Falcke H, Freund R, Friberg P, Greer CH, Gurwell MA, Ho PTP, Honma M, Inoue M, Johannsen T, Kim J, Krichbaum TP, Lamb J, León-Tavares J, Loeb A, Loinard L, MacMahon D, Marrone DP, Moran JM, Mościbrodzka M, Ortiz-León GN, Oyama T, Özel F, Plambeck RL, Pradel N, Primiani RA, Rogers AEE, Rosenfeld K, Rottmann H, Roy AL, Ruszczyk C, Smythe DL, SooHoo J, Spilker J, Stone J, Strittmatter P, Tilanus RPJ, Titus M, Vertatschitsch L, Wagner J, Wardle JFC, Weintroub J, Woody D, Wright M, Yamaguchi P, Young A, Young KH, Zensus JA, Ziurys LM (2016b) Persistent asymmetric structure of Sagittarius A* on event horizon scales. Astrophys J 820:90

Fromm CM, Ros E, Perucho M, Savolainen T, Mimica P, Kadler M, Lobanov AP, Lister ML, Kovalev YY, Zensus JA (2013) Catching the radio flare in CTA 102. II. VLBI kinematic analysis. Astron Astrophys 551:A32

Fromm CM, Perucho M, Ros E, Savolainen T, Zensus JA (2015) On the location of the supermassive black hole in CTA 102. Astron Astrophys 576:A43

Fuhrmann L, Larsson S, Chiang J, Angelakis E, Zensus JA, Nestoras I, Krichbaum TP, Ungerechts H, Sievers A, Pavlidou V, Readhead ACS, Max-Moerbeck W, Pearson TJ (2014) Detection of significant $\mathrm{cm}$ to sub-mm band radio and $\gamma$-ray correlated variability in Fermi bright blazars. Mon Not R Astron Soc 441:1899-1909

Gabuzda DC, Pushkarev AB, Cawthorne TV (2000) Analysis of $\lambda=6 \mathrm{~cm}$ VLBI polarization observations of a complete sample of northern BL Lacertae objects. Mon Not R Astron Soc 319:1109-1124

Gabuzda DC, Murray É, Cronin P (2004) Helical magnetic fields associated with the relativistic jets of four BL Lac objects. Mon Not R Astron Soc 351:L89-L93

Gebhardt K, Adams J, Richstone D, Lauer TR, Faber SM, Gültekin K, Murphy J, Tremaine S (2011) The black hole mass in M87 from Gemini/NIFS adaptive optics observations. Astrophys J 729:119

Georganopoulos M, Kazanas D (2003) Decelerating flows in TeV blazars: a resolution to the BL Lacertae-FR I unification problem. Astrophys J 594:L27-L30

Georganopoulos M, Kazanas D (2004) Witnessing the gradual slowdown of powerful extragalactic jets: the X-ray-optical-radio connection. Astrophys J 604:L81-L84

Ghisellini G, Tavecchio F (2009) Canonical high-power blazars. Mon Not R Astron Soc 397:985-1002

Ghisellini G, Tavecchio F, Chiaberge M (2005) Structured jets in TeV BL Lac objects and radiogalaxies. Implications for the observed properties. Astron Astrophys 432:401-410 
Ghisellini G, Tavecchio F, Maraschi L, Celotti A, Sbarrato T (2014) The power of relativistic jets is larger than the luminosity of their accretion disks. Nature 515:376-378

Giannios D, Spruit HC (2006) The role of kink instability in Poynting-flux dominated jets. Astron Astrophys 450:887-898

Gillessen S, Eisenhauer F, Trippe S, Alexander T, Genzel R, Martins F, Ott T (2009) Monitoring stellar orbits around the massive black hole in the galactic center. Astrophys J 692:1075-1109

Giroletti M, Giovannini G, Cotton WD, Taylor GB, Pérez-Torres MA, Chiaberge M, Edwards PG (2008) The jet of Markarian 501 from millions of Schwarzschild radii down to a few hundreds. Astron Astrophys 488:905-914

Goddi C, Falcke H, Kramer M, Rezzolla L, Brinkerink C, Bronzwaer T, Davelaar JRJ, Deane R, de Laurentis M, Desvignes G, Eatough RP, Eisenhauer F, Fraga-Encinas R, Fromm CM, Gillessen S, Grenzebach A, Issaoun S, Janßen M, Konoplya R, Krichbaum TP, Laing R, Liu K, Lu R-S, Mizuno Y, Moscibrodzka M, Müller C, Olivares H, Pfuhl O, Porth O, Roelofs F, Ros E, Schuster K, Tilanus R, Torne P, van Bemmel I, van Langevelde HJ, Wex N, Younsi Z, Zhidenko A (2017) BlackHoleCam: fundamental physics of the galactic center. Int J Mod Phys D 26:1730001-239

Gómez JL, Martí JM, Marscher AP, Ibáñez JM, Marcaide JM (1995) Parsec-scale synchrotron emission from hydrodynamic relativistic jets in active galactic nuclei. Astrophys J 449:L19

Gómez JL, Marscher AP, Alberdi A (1999) 86, 43, and 22 GHz VLBI observations of 3C 120. Astrophys J 521:L29-L32

Gómez JL, Lobanov AP, Bruni G, Kovalev YY, Marscher AP, Jorstad SG, Mizuno Y, Bach U, Sokolovsky KV, Anderson JM, Galindo P, Kardashev NS, Lisakov MM (2016) Probing the innermost regions of AGN jets and their magnetic fields with RadioAstron. I. Imaging BL Lacertae at 21 microarcsecond resolution. Astrophys J 817:96

Gopalakrishnan Nair D, Lobanov A, Ros E, Krichbaum T, Zensus A (2016) 86 GHz VLBI survey of ultra compact radio emission in active galactic nuclei. In: 41st COSPAR scientific assembly, abstracts from the meeting that was cancelled

Gubbay J, Legg AJ, Robertson DS, Moffet AT, Ekers RD, Seidel B (1969) Variations of small quasar components at 2,300 MHz. Nature 224:1094-1095

Hada K, Doi A, Kino M, Nagai H, Hagiwara Y, Kawaguchi N (2011) An origin of the radio jet in M87 at the location of the central black hole. Nature 477:185-187

Hada K, Kino M, Doi A, Nagai H, Honma M, Akiyama K, Tazaki F, Lico R, Giroletti M, Giovannini G, Orienti M, Hagiwara Y (2016) High-sensitivity 86 GHz (3.5 mm) VLBI observations of M87: deep imaging of the jet base at a resolution of 10 Schwarzschild radii. Astrophys J 817:131

Hardee PE (1979) On the configuration and propagation of jets in extragalactic radio sources. Astrophys J 234:47-55

Hardee PE (2000) On three-dimensional structures in relativistic hydrodynamic jets. Astrophys J 533:176193

Heinz S, Sunyaev RA (2003) The non-linear dependence of flux on black hole mass and accretion rate in core-dominated jets. Mon Not R Astron Soc 343:L59-L64

Helmboldt JF, Taylor GB, Tremblay S, Fassnacht CD, Walker RC, Myers ST, Sjouwerman LO, Pearson TJ, Readhead ACS, Weintraub L, Gehrels N, Romani RW, Healey S, Michelson PF, Blandford RD, Cotter G (2007) The VLBA imaging and polarimetry survey at $5 \mathrm{GHz}$. Astrophys J 658:203-216

Hirabayashi H, Hirosawa H, Kobayashi H, Murata Y, Asaki Y, Avruch IM, Edwards PG, Fomalont EB, Ichikawa T, Kii T, Okayasu R, Wajima K, Inoue M, Kawaguchi N, Chikada Y, Bushimata T, Fujisawa K, Horiuchi S, Kameno S, Miyaji T, Shibata KM, Shen Z-Q, Umemoto T, Kasuga T, Nakajima J, Takahashi Y, Enome S, Morimoto M, Ellis J, Meier DL, Murphy DW, Preston RA, Smith JG, Wietfeldt RD, Benson JM, Claussen MJ, Flatters C, Moellenbrock GA, Romney JD, Ulvestad JS, Langston GI, Minter AH, D’Addario LR, Dewdney PE, Dougherty SM, Jauncey DL, Lovell JEJ, Tingay SJ, Tzioumis AK, Taylor AR, Cannon WH, Gurvits LI, Schilizzi RT, Booth RS, Popov MV (2000) The VLBI space observatory programme and the radio-astronomical satellite HALCA. Publ Astron Soc Japan 52:955-L965

Hodgson JA, Krichbaum TP, Marscher AP, Jorstad SG, Rani B, Marti-Vidal I, Bach U, Sanchez S, Bremer M, Lindqvist M, Uunila M, Kallunki J, Vicente P, Fuhrmann L, Angelakis E, Karamanavis V, Myserlis I, Nestoras I, Chidiac C, Sievers A, Gurwell M, Zensus JA (2017) Location of $\gamma$-ray emission and magnetic field strengths in OJ 287. Astron Astrophys 597:A80

Homan DC, Kovalev YY, Lister ML, Ros E, Kellermann KI, Cohen MH, Vermeulen RC, Zensus JA, Kadler M (2006) Intrinsic brightness temperatures of AGN jets. Astrophys J 642:L115-L118 
Hovatta T, Lister ML, Aller MF, Aller HD, Homan DC, Kovalev YY, Pushkarev AB, Savolainen T (2012) MOJAVE: monitoring of jets in active galactic nuclei with VLBA experiments. VIII. Faraday rotation in parsec-scale AGN jets. Astron J 144:105

Hughes PA (2005) The origin of complex behavior of linearly polarized components in parsec-scale jets. Astrophys J 621:635-642

Hughes PA, Aller HD, Aller MF (1985) Polarized radio outbursts in Bl-Lacertae-part two-the flux and polarization of a piston-driven shock. Astrophys J 298:301

Johannsen T, Broderick AE, Plewa PM, Chatzopoulos S, Doeleman SS, Eisenhauer F, Fish VL, Genzel R, Gerhard O, Johnson MD (2016a) Testing general relativity with the shadow size of Sgr A*. Phys Rev Lett 116(3):031101

Johannsen T, Wang C, Broderick AE, Doeleman SS, Fish VL, Loeb A, Psaltis D (2016b) Testing general relativity with accretion-flow imaging of Sgr A*. Phys Rev Lett 117(9):091101

Johnson MD (2016) Stochastic optics: a scattering mitigation framework for radio interferometric imaging. Astrophys J 833:74

Johnson MD, Gwinn CR (2015) Theory and simulations of refractive substructure in resolved scatterbroadened images. Astrophys J 805:180

Johnson MD, Fish VL, Doeleman SS, Marrone DP, Plambeck RL, Wardle JFC, Akiyama K, Asada K, Beaudoin C, Blackburn L, Blundell R, Bower GC, Brinkerink C, Broderick AE, Cappallo R, Chael AA, Crew GB, Dexter J, Dexter M, Freund R, Friberg P, Gold R, Gurwell MA, Ho PTP, Honma M, Inoue M, Kosowsky M, Krichbaum TP, Lamb J, Loeb A, Lu R-S, MacMahon D, McKinney JC, Moran JM, Narayan R, Primiani RA, Psaltis D, Rogers AEE, Rosenfeld K, SooHoo J, Tilanus RPJ, Titus M, Vertatschitsch L, Weintroub J, Wright M, Young KH, Zensus JA, Ziurys LM (2015) Resolved magnetic-field structure and variability near the event horizon of Sagittarius A*. Science 350:1242-1245

Jorstad S, Marscher A (2016) The VLBA-BU-BLAZAR multi-wavelength monitoring program. Galaxies $4: 47$

Jorstad SG, Marscher AP, Mattox JR, Wehrle AE, Bloom SD, Yurchenko AV (2001) Multiepoch very long baseline array observations of EGRET-detected quasars and BL Lacertae objects: superluminal motion of gamma-ray bright blazars. Astrophys J Suppl 134:181-240

Jorstad SG, Marscher AP, Larionov VM, Agudo I, Smith PS, Gurwell M, Lähteenmäki A, Tornikoski M, Markowitz A, Arkharov AA, Blinov DA, Chatterjee R, D’Arcangelo FD, Falcone AD, Gómez JL, Hagen-Thorn VA, Jordan B, Kimeridze GN, Konstantinova TS, Kopatskaya EN, Kurtanidze O, Larionova EG, Larionova LV, McHardy IM, Melnichuk DA, Roca-Sogorb M, Schmidt GD, Skiff B, Taylor B, Thum C, Troitsky IS, Wiesemeyer H (2010) Flaring behavior of the quasar 3C 454.3 across the electromagnetic spectrum. Astrophys J 715:362-384

Junor W, Biretta JA, Livio M (1999) Formation of the radio jet in M87 at 100 Schwarzschild radii from the central black hole. Nature 401:891-892

Kadler M, Krauß F, Mannheim K, Ojha R, Müller C, Schulz R, Anton G, Baumgartner W, Beuchert T, Buson S, Carpenter B, Eberl T, Edwards PG, Eisenacher Glawion D, Elsässer D, Gehrels N, Gräfe C, Gulyaev S, Hase H, Horiuchi S, James CW, Kappes A, Kappes A, Katz U, Kreikenbohm A, Kreter M, Kreykenbohm I, Langejahn M, Leiter K, Litzinger E, Longo F, Lovell JEJ, McEnery J, Natusch T, Phillips C, Plötz C, Quick J, Ros E, Stecker FW, Steinbring T, Stevens J, Thompson DJ, Trüstedt J, Tzioumis AK, Weston S, Wilms J, Zensus JA (2016) Coincidence of a high-fluence blazar outburst with a PeV-energy neutrino event. Nat Phys 12:807-814

Karamanavis V, Fuhrmann L, Angelakis E, Nestoras I, Myserlis I, Krichbaum TP, Zensus JA, Ungerechts H, Sievers A, Gurwell MA (2016a) What can the 2008/10 broadband flare of PKS 1502+106 tell us?. Nuclear opacity, magnetic fields, and the location of $\gamma$ rays. Astron Astrophys 590:A48

Karamanavis V, Fuhrmann L, Krichbaum TP, Angelakis E, Hodgson J, Nestoras I, Myserlis I, Zensus JA, Sievers A, Ciprini S (2016b) PKS 1502+106: a high-redshift Fermi blazar at extreme angular resolution. Structural dynamics with VLBI imaging up to $86 \mathrm{GHz}$. Astron Astrophys 586:A60

Kardashev NS, Khartov VV, Abramov VV, Avdeev VY, Alakoz AV, Aleksandrov YA, Ananthakrishnan S, Andreyanov VV, Andrianov AS, Antonov NM, Artyukhov MI, Arkhipov MY, Baan W, Babakin NG, Babyshkin VE, Bartel' N, Belousov KG, Belyaev AA, Berulis JJ, Burke BF, Biryukov AV, Bubnov AE, Burgin MS, Busca G, Bykadorov AA, Bychkova VS, Vasil'kov VI, Wellington KJ, Vinogradov IS, Wietfeldt R, Voitsik PA, Gvamichava AS, Girin IA, Gurvits LI, Dagkesamanskii RD, D'Addario L, Giovannini G, Jauncey DL, Dewdney PE, D’yakov AA, Zharov VE, Zhuravlev VI, Zaslavskii GS, Zakhvatkin MV, Zinov'ev AN, Ilinen Y, Ipatov AV, Kanevskii BZ, Knorin IA, Casse JL, Kellermann 
KI, Kovalev YA, Kovalev YY, Kovalenko AV, Kogan BL, Komaev RV, Konovalenko AA, Kopelyanskii GD, Korneev YA, Kostenko VI, Kotik AN, Kreisman BB, Kukushkin AY, Kulishenko VF, Cooper DN, Kut'kin AM, Cannon WH, Larionov MG, Lisakov MM, Litvinenko LN, Likhachev SF, Likhacheva LN, Lobanov AP, Logvinenko SV, Langston G, McCracken K, Medvedev SY, Melekhin MV, Menderov AV, Murphy DW, Mizyakina TA, Mozgovoi YV, Nikolaev NY, Novikov BS, Novikov ID, Oreshko VV, Pavlenko YK, Pashchenko IN, Ponomarev YN, Popov MV, Pravin-Kumar A, Preston RA, Pyshnov VN, Rakhimov IA, Rozhkov VM, Romney JD, Rocha P, Rudakov VA, Räisänen A, Sazankov SV, Sakharov BA, Semenov SK, Serebrennikov VA, Schilizzi RT, Skulachev DP, Slysh VI, Smirnov AI, Smith JG, Soglasnov VA, Sokolovskii KV, Sondaar LH, Stepan'yants VA, Turygin MS, Turygin SY, Tuchin AG, Urpo S, Fedorchuk SD, Finkel'shtein AM, Fomalont EB, Fejes I, Fomina AN, Khapin YB, Tsarevskii GS, Zensus JA, Chuprikov AA, Shatskaya MV, Shapirovskaya NY, Sheikhet AI, Shirshakov AE, Schmidt A, Shnyreva LA, Shpilevskii VV, Ekers RD, Yakimov VE (2013) "RadioAstron"-a telescope with a size of $300000 \mathrm{~km}$ : main parameters and first observational results. Astron Rep 57:153-194

Kellermann KI, Pauliny-Toth IIK (1969) The spectra of opaque radio sources. Astrophys J 155:L71

Kellermann KI, Vermeulen RC, Zensus JA, Cohen MH (1998) Sub-milliarcsecond imaging of quasars and active galactic nuclei. Astron J 115:1295-1318

Kellermann KI, Lister ML, Homan DC, Vermeulen RC, Cohen MH, Ros E, Kadler M, Zensus JA, Kovalev YY (2004) Sub-milliarcsecond imaging of quasars and active galactic nuclei. III. Kinematics of parsecscale radio jets. Astrophys J 609:539-563

Kim J-Y, Lu R-S, Krichbaum T, Bremer M, Zensus J, Walker R and the M87 collaboration (2016) Resolving the base of the relativistic jet in M87 at $6 R_{\text {sch }}$ resolution with global mm-VLBI. Galaxies 4:39

Koide S, Shibata K, Kudoh T (1999) Relativistic jet formation from black hole magnetized accretion disks: method, tests, and applications of a general RelativisticMagnetohydrodynamic numerical code. Astrophys J 522:727-752

Komissarov S (2012) Central engines: acceleration, collimation and confinement of jets. In: Boettcher M, Harris DE, Krawczynski H (eds) Relativistic jets from active galactic nuclei. Wiley, Berlin, pp 81-114

Komissarov SS, Barkov MV, Vlahakis N, Königl A (2007) Magnetic acceleration of relativistic active galactic nucleus jets. Mon Not R Astron Soc 380:51-70

Königl A (1981) Relativistic jets as X-ray and gamma-ray sources. Astrophys J 243:700-709

Kovalev YY, Lister ML, Homan DC, Kellermann KI (2007) The inner jet of the radio galaxy M87. Astrophys J 668:L27-L30

Kovalev YY, Kardashev NS, Kellermann KI, Lobanov AP, Johnson MD, Gurvits LI, Voitsik PA, Zensus JA, Anderson JM, Bach U, Jauncey DL, Ghigo F, Ghosh T, Kraus A, Kovalev YA, Lisakov MM, Petrov LY, Romney JD, Salter CJ, Sokolovsky KV (2016) RadioAstron observations of the quasar 3C273: a challenge to the brightness temperature limit. Astrophys J 820:L9

Koyama S, Kino M, Giroletti M, Doi A, Giovannini G, Orienti M, Hada K, Ros E, Niinuma K, Nagai H, Savolainen T, Krichbaum TP, Pérez-Torres MÁ (2016) Discovery of off-axis jet structure of TeV blazar Mrk 501 with mm-VLBI. Astron Astrophys 586:A113

Krichbaum TP, Zensus JA, Witzel A, Mezger PG, Standke KJ, Schalinski CJ, Alberdi A, Marcaide JM, Zylka R, Rogers AEE, Booth RS, Ronnang BO, Colomer F, Bartel N, Shapiro II (1993) First 43-GHZ VLBI detection of the compact source Sgr A* in the Galactic Center. Astron Astrophys 274:L37

Krichbaum TP, Graham DA, Greve A, Wink JE, Alcolea J, Colomer F, de Vicente P, Baudry A, GomezGonzalez J, Grewing M, Witzel A (1997) 215 GHz VLBI observations of bright active galactic nuclei. Astron Astrophys 323:L17-L20

Krichbaum TP, Graham DA, Witzel A, Greve A, Wink JE, Grewing M, Colomer F, de Vicente P, GomezGonzalez J, Baudry A, Zensus JA (1998) VLBI observations of the galactic center source Sgr A* at $86 \mathrm{GHz}$ and $215 \mathrm{GHz}$. Astron Astrophys 335:L106-L110

Krichbaum TP, Agudo I, Bach U, Witzel A, Zensus JA (2006) VLBI at the highest frequencies_AGN studies with micro-arcsecond resolution. In: Proceedings of the 8th European VLBI network symposium, PoS(8thEVN)002

Krichbaum TP, Roy A, Lu R-S, Zensus JA, Fish V, Doeleman S, Event Horizon Telescope (EHT) Collaboration (2014) Millimiter VLBI observations: black hole physics and the origin of jets. In: Proceedings of the 12th European VLBI network symposium and users meeting (EVN 2014), 7-10 October 2014, Cagliari, Italy. id.13, p 13

Lähteenmäki A, Valtaoja E (2003) Testing of inverse Compton models for active galactic nuclei with gamma-ray and radio observations. Astrophys J 590:95-108 
Laing RA (1980) A model for the magnetic-field structure in extended radio sources. Mon Not R Astron Soc 193:439-449

Lee S-S, Lobanov AP, Krichbaum TP, Witzel A, Zensus A, Bremer M, Greve A, Grewing M (2008) A global 86 GHz VLBI survey of compact radio sources. Astron J 136:159-180

Lee S-S, Lobanov AP, Krichbaum TP, Zensus JA (2016) Acceleration of compact radio jets on sub-parsec scales. Astrophys J 826:135

León-Tavares J, Lobanov AP, Chavushyan VH, Arshakian TG, Doroshenko VT, Sergeev SG, Efimov YS, Nazarov SV (2010) Relativistic plasma as the dominant source of the optical continuum emission in the broad-line radio galaxy 3C 120. Astrophys J 715:355-361

León-Tavares J, Valtaoja E, Tornikoski M, Lähteenmäki A, Nieppola E (2011) The connection between gamma-ray emission and millimeter flares in Fermi/LAT blazars. Astron Astrophys 532:A146

Lico R, Giroletti M, Orienti M, Gómez JL, Casadio C, D’Ammando F, Blasi MG, Cotton W, Edwards PG, Fuhrmann L, Jorstad S, Kino M, Kovalev YY, Krichbaum TP, Marscher AP, Paneque D, Piner BG, Sokolovsky KV (2014) Very long baseline polarimetry and the $\gamma$-ray connection in Markarian 421 during the broadband campaign in 2011. Astron Astrophys 571:A54

Lisakov MM, Kovalev YY, Savolainen T, Hovatta T, Kutkin AM (2017) A connection between $\gamma$-ray and parsec-scale radio flares in the blazar 3C 273. Mon Not R Astron Soc 468:4478-4493

Lister ML, Homan DC (2005) MOJAVE: monitoring of jets in active galactic nuclei with VLBA experiments. I. First-epoch $15 \mathrm{GHz}$ linear polarization images. Astron J 130:1389-1417

Lister ML, Marscher AP (1997) Statistical effects of doppler beaming and Malmquist bias on flux-limited samples of compact radio sources. Astrophys J 476:572-588

Lister ML, Kellermann KI, Vermeulen RC, Cohen MH, Zensus JA, Ros E (2003) 4C +12.50: a superluminal precessing jet in the recent merger system IRAS 13451+1232. Astrophys J 584:135-146

Lister ML, Aller HD, Aller MF, Cohen MH, Homan DC, Kadler M, Kellermann KI, Kovalev YY, Ros E, Savolainen T, Zensus JA, Vermeulen RC (2009) MOJAVE: monitoring of jets in active galactic nuclei with VLBA experiments. V. Multi-epoch VLBA images. Astron J 137:3718-3729

Lister ML, Aller MF, Aller HD, Homan DC, Kellermann KI, Kovalev YY, Pushkarev AB, Richards JL, Ros E, Savolainen T (2016) MOJAVE: XIII. Parsec-scale AGN jet kinematics analysis based on 19 years of VLBA observations at $15 \mathrm{GHz}$. Astron J 152:12

Lobanov AP (1998) Ultracompact jets in active galactic nuclei. Astron Astrophys 330:79-89

Lobanov AP, Zensus JA (2001) A cosmic double helix in the archetypical quasar 3C273. Science 294:128131

Lobanov AP, Krichbaum TP, Graham DA, Witzel A, Kraus A, Zensus JA, Britzen S, Greve A, Grewing M (2000) 86 GHz VLBI survey of compact radio sources. Astron Astrophys 364:391-408

Lonsdale CJ, Doeleman SS, Phillips RB (1998) A 3 millimeter VLBI continuum source survey. Astron J 116:8-12

Lu R-S, Krichbaum TP, Eckart A, König S, Kunneriath D, Witzel G, Witzel A, Zensus JA (2011) Multiwavelength VLBI observations of Sagittarius A*. Astron Astrophys 525:A76

Lu R-S, Fish VL, Akiyama K, Doeleman SS, Algaba JC, Bower GC, Brinkerink C, Chamberlin R, Crew G, Cappallo RJ, Dexter M, Freund R, Friberg P, Gurwell MA, Ho PTP, Honma M, Inoue M, Jorstad SG, Krichbaum TP, Loinard L, MacMahon D, Marrone DP, Marscher AP, Moran JM, Plambeck R, Pradel N, Primiani R, Tilanus RPJ, Titus M, Weintroub J, Wright M, Young KH, Ziurys LM (2013) Fine-scale structure of the quasar 3C 279 measured with $1.3 \mathrm{~mm}$ very long baseline interferometry. Astrophys J 772:13

Lu R-S, Roelofs F, Fish VL, Shiokawa H, Doeleman SS, Gammie CF, Falcke H, Krichbaum TP, Zensus JA (2016) Imaging an event horizon: mitigation of source variability of Sagittarius A*. Astrophys J $817: 173$

Luminet J-P (1979) Image of a spherical black hole with thin accretion disk. Astron Astrophys 75:228-235

Ly C, Walker RC, Wrobel JM (2004) An attempt to probe the radio jet collimation regions in NGC 4278, NGC 4374 (M84), and NGC 6166. Astron J 127:119-124

Ly C, Walker RC, Junor W (2007) High-frequency VLBI imaging of the jet base of M87. Astrophys J 660:200-205

Lynden-Bell D (1969) Galactic nuclei as collapsed old quasars. Nature 223:690-694

Lyubarsky Y (2009) Asymptotic structure of Poynting-dominated jets. Astrophys J 698:1570-1589

Lyutikov M, Pariev VI, Gabuzda DC (2005) Polarization and structure of relativistic parsec-scale AGN jets. Mon Not R Astron Soc 360:869-891 
Mannheim K, Biermann PL (1992) Gamma-ray flaring of 3C 279—a proton-initiated cascade in the jet? Astron Astrophys 253:L21-L24

Marcaide JM, Pauliny-Toth IIK, Graham DA, Ronnang B, Booth RS (1985) Millimeter-wavelength VLBIfirst results from intercontinental observations with a resolution of 0.2 milliarcseconds. In: Shaver PA, Kjar K (eds) European southern observatory conference and workshop proceedings, vol 22, pp 157167

Maraschi L, Ghisellini G, Celotti A (1992) A jet model for the gamma-ray emitting blazar 3C 279. Astrophys J 397:L5-L9

Marcaide JM, Shapiro II (1984) VLBI study of 1038 + 528 A and B-discovery of wavelength dependence of peak brightness location. Astrophys J 276:56-59

Marscher AP (1996) The inner jets of blazars. In: Hardee PE, Bridle AH, Zensus JA (eds) Energy transport in radio galaxies and quasars, Astronomical Society of the Pacific conference series, vol 100, p 45

Marscher AP (2005) Multiband impressions of active galactic nuclei. Mem Soc Astron Ital 76:168

Marscher AP (2006) Relativistic jets in active galactic nuclei. In: Hughes PA, Bregman JN (eds) Relativistic jets: the common physics of AGN, microquasars, and gamma-ray bursts. American Institute of physics conference series, vol 856, pp 1-22

Marscher AP (2008) The core of a blazar jet. In: Rector TA, De Young DS (eds) Extragalactic jets: theory and observation from radio to gamma ray. Astronomical Society of the Pacific conference series, vol $386, \mathrm{p} 437$

Marscher AP (2009) The compact structure of blazars at high frequencies. In: Hagiwara Y, Fomalont E, Tsuboi M, Yasuhiro M (eds) Approaching micro-arcsecond resolution with VSOP-2: astrophysics and technologies. Astronomical Society of the Pacific conference series, vol 402, p 194

Marscher AP (2011) Astrophysics: broad escape from the abyss. Nature 477:164-165

Marscher AP, Gear WK (1985) Models for high-frequency radio outbursts in extragalactic sources, with application to the early 1983 millimeter-to-infrared flare of 3C 273. Astrophys J 298:114-127

Marscher AP, Jorstad SG (2010) Rapid variability of gamma-ray emission from sites near the $43 \mathrm{GHz}$ cores of blazar jets. arXiv e-prints arXiv:1005.5551

Marscher AP, Jorstad SG, D’ Arcangelo FD, Smith PS, Williams GG, Larionov VM, Oh H, Olmstead AR, Aller MF, Aller HD, McHardy IM, Lähteenmäki A, Tornikoski M, Valtaoja E, Hagen-Thorn VA, Kopatskaya EN, Gear WK, Tosti G, Kurtanidze O, Nikolashvili M, Sigua L, Miller HR, Ryle WT (2008) The inner jet of an active galactic nucleus as revealed by a radio-to- $\gamma$-ray outburst. Nature 452:966-969

Marscher AP, Jorstad SG, Larionov VM, Aller MF, Aller HD, Lähteenmäki A, Agudo I, Smith PS, Gurwell M, Hagen-Thorn VA, Konstantinova TS, Larionova EG, Larionova LV, Melnichuk DA, Blinov DA, Kopatskaya EN, Troitsky IS, Tornikoski M, Hovatta T, Schmidt GD, D’Arcangelo FD, Bhattarai D, Taylor B, Olmstead AR, Manne-Nicholas E, Roca-Sogorb M, Gómez JL, McHardy IM, Kurtanidze O, Nikolashvili MG, Kimeridze GN, Sigua LA (2010) Probing the inner jet of the quasar PKS 1510-089 with multi-waveband monitoring during strong gamma-ray activity. Astrophys J 710:L126-L131

Martí-Vidal I, Krichbaum TP, Marscher A, Alef W, Bertarini A, Bach U, Schinzel FK, Rottmann H, Anderson JM, Zensus JA, Bremer M, Sanchez S, Lindqvist M, Mujunen A (2012) On the calibration of fullpolarization $86 \mathrm{GHz}$ global VLBI observations. Astron Astrophys 542:A107

Martí-Vidal I, Muller S, Vlemmings W, Horellou C, Aalto S (2015) A strong magnetic field in the jet base of a supermassive black hole. Science 348:311-314

Martí-Vidal I, Roy A, Conway J, Zensus AJ (2016) Calibration of mixed-polarization interferometric observations. Tools for the reduction of interferometric data from elements with linear and circular polarization receivers. Astron Astrophys 587:A143

Matthews LD, Alef W, Anderson J, Barkats D, Crew GB, Doeleman SS, Fish VL, Greenberg J, Hecht MH, Hiriart R, Honma M, Impellizzeri C, Inoue M, Lacasse R, Lopez B, Mora-Klein M, Nagar N, Pankratius V, Pradel N, Rottmann H, Roy A, Ruszczyk C, Saez A, Shillue B, Treacy R, ALMA Phasing Project Team (2014) The ALMA phasing project: new frontiers in ultra-high resolution astronomy enabled by a beamformed ALMA. In: American Astronomical Society meeting abstracts, vol 223, p 443.02

McKinney JC (2006) General relativistic magnetohydrodynamic simulations of the jet formation and largescale propagation from black hole accretion systems. Mon Not R Astron Soc 368:1561-1582

McKinney JC, Blandford RD (2009) Stability of relativistic jets from rotating, accreting black holes via fully three-dimensional magnetohydrodynamic simulations. Mon Not R Astron Soc 394:L126-L130 
Meier DL (2003) The theory and simulation of relativistic jet formation: towards a unified model for microand macroquasars. New Astron Rev 47:667-672

Meier DL (2012) Black hole astrophysics: the engine paradigm. Springer, Heidelberg

Meier DL, Koide S, Uchida Y (2001) Magnetohydrodynamic production of relativistic jets. Science 291:8492

Merloni A, Heinz S, di Matteo T (2003) A fundamental plane of black hole activity. Mon Not RAS 345:10571076

Mertens F, Lobanov AP, Walker RC, Hardee PE (2016) Kinematics of the jet in M 87 on scales of 100-1000 Schwarzschild radii. Astron Astrophys 595:A54

Mignone A, Rossi P, Bodo G, Ferrari A, Massaglia S (2010) High-resolution 3D relativistic MHD simulations of jets. Mon Not R Astron Soc 402:7-12

Molina SN, Agudo I, Gómez JL, Krichbaum TP, Martí-Vidal I, Roy AL (2014) Evidence of internal rotation and a helical magnetic field in the jet of the quasar NRAO 150. Astron Astrophys 566:A26

Morozova DA, Larionov VM, Troitsky IS, Jorstad SG, Marscher AP, Gómez JL, Blinov DA, Efimova NV, Hagen-Thorn VA, Hagen-Thorn EI, Joshi M, Konstantinova TS, Kopatskaya EN, Larionova LV, Larionova EG, Lähteenmäki A, Tammi J, Rastorgueva-Foi E, McHardy I, Tornikoski M, Agudo I, Casadio C, Molina SN, Volvach AE, Volvach LN (2014) The outburst of the blazar S4 0954+658 in 2011 March-April. Astron J 148:42

Mościbrodzka M, Gammie CF, Dolence JC, Shiokawa H, Leung PK (2009) Radiative models of Sgr A* from GRMHD simulations. Astrophys J 706:497-507

Nagai H, Orienti M, Kino M, Suzuki K, Giovannini G, Doi A, Asada K, Giroletti M, Kataoka J, D’ Ammando F, Inoue M, Lähteenmäki A, Tornikoski M, León-Tavares J, Kameno S, Bach U (2012) VLBI and single-dish monitoring of 3C 84 for the period 2009-2011. Mon Not R Astron Soc 423:L122-L126

Nagai H, Haga T, Giovannini G, Doi A, Orienti M, D’Ammando F, Kino M, Nakamura M, Asada K, Hada K, Giroletti M (2014) Limb-brightened jet of 3C 84 revealed by the $43 \mathrm{GHz}$ very-long-baseline-array observation. Astrophys J 785:53

Narayan R, Yi I (1994) Advection-dominated accretion: a self-similar solution. Astrophys J 428:L13-L16

Narayan R, Igumenshchev IV, Abramowicz MA (2003) Magnetically arrested disk: an energetically efficient accretion flow. Publ Astron Soc Japan 55:L69-L72

Neumayer N (2010) The supermassive black hole at the heart of Centaurus A: revealed by the kinematics of gas and stars. PASA 27:449-456

Ortiz-León GN, Johnson MD, Doeleman SS, Blackburn L, Fish VL, Loinard L, Reid MJ, Castillo E, Chael AA, Hernández-Gómez A, Hughes DH, León-Tavares J, Lu R-S, Montaña A, Narayanan G, Rosenfeld K, Sánchez D, Schloerb FP, Shen Z-q, Shiokawa H, SooHoo J, Vertatschitsch L (2016) The intrinsic shape of Sagittarius A* at $3.5 \mathrm{~mm}$ wavelength. Astrophys J 824:40

O'Sullivan SP, Gabuzda DC (2009) Three-dimensional magnetic field structure of six parsec-scale active galactic nuclei jets. Mon Not R Astron Soc 393:429-456

Pacholczyk AG (1970) Radio astrophysics. Nonthermal processes in galactic and extragalactic sources. Freeman, San Francisco

Padin S, Woody DP, Hodges MW, Rogers AEE, Emerson DT, Jewell PR, Lamb J, Perfetto A, Wright MCH (1990) 223 GHz VLBI observations of 3C 273. Astrophys J 360:L11-L13

Padovani P, Resconi E, Giommi P, Arsioli B, Chang YL (2016) Extreme blazars as counterparts of IceCube astrophysical neutrinos. Mon Not R Astron Soc 457:3582-3592

Perley RA, Dreher JW, Cowan JJ (1984) The jet and filaments in Cygnus A. Astrophys J 285:L35-L38

Perucho M, Kovalev YY, Lobanov AP, Hardee PE, Agudo I (2012) Anatomy of helical extragalactic jets: the case of S5 0836+710. Astrophys J 749:55

Piner BG, Pant N, Edwards PG (2010) The jets of TeV blazars at higher resolution: $43 \mathrm{GHz}$ and polarimetric VLBA observations from 2005 to 2009. Astrophys J 723:1150-1167

Polko P, Meier DL, Markoff S (2010) Determining the optimal locations for shock acceleration in magnetohydrodynamical jets. Astrophys J 723:1343-1350

Pollack LK, Taylor GB, Zavala RT (2003) VLBI polarimetry of 177 sources from the Caltech-Jodrell bank flat-spectrum survey. Astrophys J 589:733-751

Poutanen J, Stern B (2010) GeV breaks in blazars as a result of gamma-ray absorption within the broad-line region. Astrophys J 717:L118-L121

Pushkarev AB, Kovalev YY, Lister ML, Savolainen T (2009) Jet opening angles and gamma-ray brightness of AGN. Astron Astrophys 507:L33-L36 
Pushkarev AB, Kovalev YY, Lister ML (2010) Radio/gamma-ray time delay in the parsec-scale cores of active galactic nuclei. Astrophys J 722:L7-L11

Pushkarev AB, Hovatta T, Kovalev YY, Lister ML, Lobanov AP, Savolainen T, Zensus JA (2012) MOJAVE: monitoring of jets in active galactic nuclei with VLBA experiments. IX. Nuclear opacity. Astron Astrophys 545:A113

Ramakrishnan V, León-Tavares J, Rastorgueva-Foi EA, Wiik K, Jorstad SG, Marscher AP, Tornikoski M, Agudo I, Lähteenmäki A, Valtaoja E, Aller MF, Blinov DA, Casadio C, Efimova NV, Gurwell MA, Gómez JL, Hagen-Thorn VA, Joshi M, Järvelä E, Konstantinova TS, Kopatskaya EN, Larionov VM, Larionova EG, Larionova LV, Lavonen N, MacDonald NR, McHardy IM, Molina SN, Morozova DA, Nieppola E, Tammi J, Taylor BW, Troitsky IS (2014) The connection between the parsec-scale radio jet and $\gamma$-ray flares in the blazar 1156+295. Mon Not R Astron Soc 445:1636-1646

Ramakrishnan V, Hovatta T, Nieppola E, Tornikoski M, Lähteenmäki A, Valtaoja E (2015) Locating the $\gamma$-ray emission site in Fermi/LAT blazars from correlation analysis between $37 \mathrm{GHz}$ radio and $\gamma$-ray light curves. Mon Not R Astron Soc 452:1280-1294

Rani B, Krichbaum TP, Marscher AP, Jorstad SG, Hodgson JA, Fuhrmann L, Zensus JA (2014) Jet outflow and gamma-ray emission correlations in S5 0716+714. Astron Astrophys 571:L2

Rantakyrö FT, Baath LB, Backer DC, Booth RS, Carlstrom JE, Emerson DT, Grewing M, Hirabayashi H, Hodges MW, Inoue M, Kobayashi H, Krichbaum TP, Kus AJ, Moran JM, Morimoto M, Padin S, Plambeck RL, Predmore R, Rogers AEE, Schalinski C, Witzel A, Woody D, Wright MCH, Zensus A (1998) 50 MU as resolution VLBI images of AGN's at lambda $3 \mathrm{~mm}$. Astron Astrophys Suppl 131:451-467

Rauch C, Ros E, Krichbaum TP, Eckart A, Zensus JA, Shahzamanian B, Mužić K (2016) Wisps in the galactic center: near-infrared triggered observations of the radio source Sgr A* at $43 \mathrm{GHz}$. Astron Astrophys 587:A37

Readhead ACS (1994) Equipartition brightness temperature and the inverse Compton catastrophe. Astrophys J 426:51-59

Readhead ACS, Mason CR, Mofett AT, Pearson TJ, Seielstad GA, Woody DP, Backer DC, Plambeck RL, Welch WJ, Wright MCH, Rogers AEE, Webber JC, Shapiro II, Moran JM, Goldsmith PF, Predmore CR, Baath L, Ronnang B (1983) Very long baseline interferometry at a wavelength of $3.4 \mathrm{~mm}$. Nature 303:504-506

Rees MJ (1966) Appearance of relativistically expanding radio sources. Nature 211:468-470

Rees MJ (1970) Extragalactic variable radio sources. Nature 227:1303-1306

Reid MJ, Menten KM, Brunthaler A, Zheng XW, Dame TM, Xu Y, Wu Y, Zhang B, Sanna A, Sato M, Hachisuka K, Choi YK, Immer K, Moscadelli L, Rygl KLJ, Bartkiewicz A (2014) Trigonometric parallaxes of high mass star forming regions: the structure and kinematics of the Milky way. Astrophys J 783:130

Reynolds CS, Fabian AC, Celotti A, Rees MJ (1996) The matter content of the jet in M87: evidence for an electron-positron jet. Mon Not R Astron Soc 283:873-880

Rogers AEE, Moran JM Jr (1981) Coherence limits for very-long-baseline interferometry. IEEE Trans Instrum Meas 30:283-286

Rogers AEE, Moffet AT, Backer DC, Moran JM (1984) Coherence limits in VLBI observations at 3millimeter wavelength. Radio Sci 19:1552-1560

Rogers AEE, Phillips RB, Lonsdale CJ (1995) A coordinated millimeter-VLBI array (CMVA). In: American Astronomical Society meeting abstracts, vol 27. Bulletin of the American Astronomical Society, p 1300

Ryle M, Hewish A (1960) The synthesis of large radio telescopes. Mon Not R Astron Soc 120:220

Ryle M, Vonberg DD (1946) Solar radiation on 175 Mc./s. Nature 158:339-340

Salpeter EE (1964) Accretion of interstellar matter by massive objects. Astrophys J 140:796-800

Sams BJ, Eckart A, Sunyaev R (1996) Near-infrared jets in the Galactic microquasar GRS1915+105. Nature 382:47-49

Scharwächter J, McGregor PJ, Dopita MA, Beck TL (2013) Kinematics and excitation of the molecular hydrogen accretion disc in NGC 1275. Mon Not R Astron Soc 429:2315-2332

Scheuer PAG, Readhead ACS (1979) Superluminally expanding radio sources and the radio-quiet QSOs. Nature 277:182-185

Schinzel FK, Lobanov AP, Taylor GB, Jorstad SG, Marscher AP, Zensus JA (2012) Relativistic outflow drives $\gamma$-ray emission in 3C 345. Astron Astrophys 537:A70

Schmidt M (1963) 3C 273: a star-like object with large red-shift. Nature 197:1040 
Sikora M, Begelman MC, Rees MJ (1994) Comptonization of diffuse ambient radiation by a relativistic jet: the source of gamma rays from blazars? Astrophys J 421:153-162

Sikora M, Moderski R, Madejski GM (2008) 3C 454.3 reveals the structure and physics of its "blazar zone". Astrophys J 675:71-78

Sikora M, Stawarz Ł, Moderski R, Nalewajko K, Madejski GM (2009) Constraining emission models of luminous blazar sources. Astrophys J 704:38-50

Sironi L, Petropoulou M, Giannios D (2015) Relativistic jets shine through shocks or magnetic reconnection? Mon Not R Astron Soc 450:183-191

Sol H, Pelletier G, Asseo E (1989) Two-flow model for extragalactic radio jets. Mon Not R Astron Soc 237:411-429

Spruit HC (2010) Theory of magnetically powered jets. In: Belloni T (ed) Lecture notes in physics, vol 794. Springer, Berlin, p 233

Spruit HC, Foglizzo T, Stehle R (1997) Collimation of magnetically driven jets from accretion discs. Mon Not R Astron Soc 288:333-342

Suzuki K, Nagai H, Kino M, Kataoka J, Asada K, Doi A, Inoue M, Orienti M, Giovannini G, Giroletti M, Lähteenmäki A, Tornikoski M, León-Tavares J, Bach U, Kameno S, Kobayashi H (2012) Exploring the central sub-parsec region of the $\gamma$-ray bright radio galaxy $3 \mathrm{C} 84$ with VLBA at $43 \mathrm{GHz}$ in the period of 2002-2008. Astrophys J 746:140

Tadhunter C, Marconi A, Axon D, Wills K, Robinson TG, Jackson N (2003) Spectroscopy of the nearnuclear regions of Cygnus A: estimating the mass of the supermassive black hole. Mon Not R Astron Soc 342:861-875

Tateyama CE, Kingham KA (2004) Structure of OJ 287 from geodetic VLBA Data. Astrophys J 608:149_ 156

Tavani M, Barbiellini G, Argan A, Bulgarelli A, Caraveo P, Chen A, Cocco V, Costa E, de Paris G, Del Monte E, Di Cocco G, Donnarumma I, Feroci M, Fiorini M, Froysland T, Fuschino F, Galli M, Gianotti F, Giuliani A, Evangelista Y, Labanti C, Lapshov I, Lazzarotto F, Lipari P, Longo F, Marisaldi M, Mastropietro M, Mauri F, Mereghetti S, Morelli E, Morselli A, Pacciani L, Pellizzoni A, Perotti F, Picozza P, Pontoni C, Porrovecchio G, Prest M, Pucella G, Rapisarda M, Rossi E, Rubini A, Soffitta P, Trifoglio M, Trois A, Vallazza E, Vercellone S, Zambra A, Zanello D, Giommi P, Antonelli A, Pittori C (2008) The AGILE space mission. Nucl Instrum Methods Phys Res A 588:52-62

Tavecchio F, Ghisellini G (2014) On the spine-layer scenario for the very high-energy emission of NGC 1275. Mon Not R Astron Soc 443:1224-1230

Tavecchio F, Ghisellini G, Bonnoli G, Ghirlanda G (2010) Constraining the location of the emitting region in Fermi blazars through rapid $\gamma$-ray variability. Mon Not R Astron Soc 405:L94-L98

Tavecchio F, Ghisellini G, Guetta D (2014) Structured jets in BL Lac objects: efficient PeV neutrino factories? Astrophys J 793:L18

Taylor GB (1998) Magnetic fields in quasar cores. Astrophys J 506:637-646

Taylor GB, Zavala R (2010) Are there rotation measure gradients across active galactic nuclei jets? Astrophys J 722:L183-L187

Tchekhovskoy A (2015) Launching of active galactic nuclei jets. In: Contopoulos I, Gabuzda D, Kylafis N (eds) The formation and disruption of black hole jets. Astrophysics and Space Science Library, vol 414. Springer, Cham, p 45

Tchekhovskoy A, Bromberg O (2016) Three-dimensional relativistic MHD simulations of active galactic nuclei jets: magnetic kink instability and Fanaroff-Riley dichotomy. Mon Not R Astron Soc 461:L46L50

Tchekhovskoy A, Narayan R, McKinney JC (2011) Efficient generation of jets from magnetically arrested accretion on a rapidly spinning black hole. Mon Not R Astron Soc 418:L79-L83

Thompson DJ, Bertsch DL, Fichtel CE, Hartman RC, Hofstadter R, Hughes EB, Hunter SD, Hughlock BW, Kanbach G, Kniffen DA, Lin YC, Mattox JR, Mayer-Hasselwander HA, von Montigny C, Nolan PL, Nel HI, Pinkau K, Rothermel H, Schneid EJ, Sommer M, Sreekumar P, Tieger D, Walker AH (1993) Calibration of the energetic gamma-ray experiment telescope (EGRET) for the Compton gamma-ray observatory. Astrophys J Suppl 86:629-656

Thompson AR, Moran JM, Jr Swenson GW (2017) Interferometry and synthesis in radio astronomy, 3rd edn. Springer, Cham

Tuccari G, Comoretto G, Melis A, Buttaccio S (2012) The DBBC environment for millimeter radioastronomy. In: Millimeter, submillimeter, and far-infrared detectors and instrumentation for astronomy VI. Proceedings of SPIE, vol 8452, p 84522W 
Turland BD, Scheuer PAG (1976) Instabilities of Kelvin-Helmholtz type for relativistic streaming. Mon Not R Astron Soc 176:421-441

Ulaby F, Long D, Blackwell W, Elachi C, Sarabandi K (2014) Microwave radar and radiometric remote sensing. University of Michigan Press, Ann Arbor

Urry CM, Padovani P (1995) Unified schemes for radio-loud active galactic nuclei. Publ Astron Soc Pac 107:803

Valtaoja E, Teraesranta H (1996) The relationship between gamma emission and radio flares in AGN. Astron Astrophys Suppl 120:491-494

Valtaoja E, Terasranta H (1995) Gamma radiation from radio shocks in AGN jets. Astron Astrophys 297:L13-L16

Vlahakis N, Königl A (2003) Relativistic magnetohydrodynamics with application to gamma-ray burst outflows. I. Theory and semianalytic trans-Alfvénic solutions. Astrophys J 596:1080-1103

Vlahakis N, Königl A (2004) Magnetic driving of relativistic outflows in active galactic nuclei. I. Interpretation of parsec-scale accelerations. Astrophys J 605:656-661

Wagner J, Roy AL, Krichbaum TP, Alef W, Bansod A, Bertarini A, Güsten R, Graham D, Hodgson J, Märtens R, Menten K, Muders D, Rottmann H, Tuccari G, Weiss A, Wieching G, Wunderlich M, Zensus JA, Araneda JP, Arriagada O, Cantzler M, Duran C, Montenegro-Montes FM, Olivares R, Caro P, Bergman P, Conway J, Haas R, Johansson J, Lindqvist M, Olofsson H, Pantaleev M, Buttaccio S, Cappallo R, Crew G, Doeleman S, Fish V, Lu R-S, Ruszczyk C, SooHoo J, Titus M, Freund R, Marrone D, Strittmatter P, Ziurys L, Blundell R, Primiani R, Weintroub J, Young K, Bremer M, Sánchez S, Marscher AP, Chilson R, Asada K, Inoue M (2015) First 230 GHz VLBI fringes on 3C 279 using the APEX telescope. Astron Astrophys 581:A32

Walker RC, Hardee PE, Davies F, Ly C, Junor W, Mertens F, Lobanov A (2016) Observations of the structure and dynamics of the inner M87 jet. Galaxies 4:46

Woo J-H, Urry CM (2002) Active galactic nucleus black hole masses and bolometric luminosities. Astrophys J 579:530-544

Yuan F, Markoff S, Falcke H (2002) A jet-ADAF model for Sgr A*. Astron Astrophys 383:854-863

Zamaninasab M, Savolainen T, Clausen-Brown E, Hovatta T, Lister ML, Krichbaum TP, Kovalev YY, Pushkarev AB (2013) Evidence for a large-scale helical magnetic field in the quasar 3C 454.3. Mon Not R Astron Soc 436:3341-3356

Zamaninasab M, Clausen-Brown E, Savolainen T, Tchekhovskoy A (2014) Dynamically important magnetic fields near accreting supermassive black holes. Nature 510:126-128

Zavala RT, Taylor GB (2004) A view through Faraday's fog. II. Parsec-scale rotation measures in 40 active galactic nuclei. Astrophys J 612:749-779

Zensus JA (1997) Parsec-scale jets in extragalactic radio sources. Annu Rev Astron Astrophys 35:607-636 